

\section{TAPPI Paper and Board (6 times per year)}

Bursting strength

Tearing strength

Tensile breaking strength

Elongation to break

Tensile energy absorption

Folding endurance

stiffness

Air resistance

Grammage

\section{Smoothness}

Surface pick strength

$\mathrm{K} \& \mathrm{~N}$ ink absorption

$\mathrm{pH}$

Opacity

Blue reflectance (brightness)

Specular gloss, $75^{\circ}$

Thickness

Concora (flat crush)

Ring crush

FKBG-API Containerboard (48 times per year)

Mullen burst of linerboard

Concora test of medium

MCCA Color and Appearance (4 times per year)

Gloss at $60^{\circ}$

Color and color difference

Retroreflectivity

Rubber ( 4 times per year)

Tensile strength, ultimate elongation and tensile stress

Hardness

Mooney viscosity

Vulcanization properties

ASTM Textiles ( 3 times per year)

Flammability (FF3-71 and FF5-74)

ASTM Cement ( 2 times per year)

Chemical ( 11 chemical components)

Physical ( 8 characteristics)

AASHTO Bituminous

Asphalt cement (2 times per year)

Cutbacks (once a year)

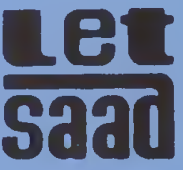

Collaborative Reference Programs B360 Polymer Building National Bureau of Standards Washington, D.C. 20234 


\title{
COLLABORATIVE REFERENCE PROGRAM FOR COLOR AND APPEARANCE
}

COLOR AND COLOR DIFFERENCE

\author{
J. Horlick \\ Office of Testing Laboratory \\ Evaluation Technology, \\ Office of Engineering Standards, \\ National Engineering Laboratory \\ T.L. Cummings \\ NBS Research Associate \\ Collaborative Testing \\ Services, Inc.
}

\section{U. S. DEPARTMENT OF COMMERCE}

National Bureau of Standards 



\section{INTRODUCTION}

This Collaborative Reference Program is sponsored by the Manufacturers Council on Color and Appearance and the National Bureau of Standards. Four times per year, color chip samples are distributed to each participating laboratory. After the data has been returned to and analyzed by NBS, a report (as illustrated by this report) showing the data from all participants is prepared.

Reflectance values for 40 wavelengths and colorimetric data for $45 / 0$ reflectance factor have been provided by NBS. For further explanation, see page vi. A plot of the spectrophotometric curves of the samples was provided by Hemmendinger Color Laboratory, Belvedere, New Jersey. The NBS Reflectance values have succeeded the tentative values which were given in previous reports.

If there are any questions on the notes, the analyses, or the report in general, contact J. Horlick on 301-921-2946.

April 20, 1979 
TABLE OF CONTENTS

Page

Introduction

Table of Contents

Explanation of WHITE Sample Data

iv

$\triangle E$ Calculations

v

Special Instrument Identification

vi

Notes on NBS Reflectance Values

Key to Tables

Analysis of laboratories in $X, Y, Z$ space, normal data, samples C91-C92

Analysis of laboratories in $X, Y, Z$ space, normal data, samples C93-C94

Analys is of laboratories in L, a, b space, normal data, samples C91-C92

Analysis of laboratories in $L, a, b$ space, normal data, samples C93-C94

Ratios of laboratories in X, Y, Z space, WHITE sample

Ratios of laboratories in L, a, b space, WHITE sample (results according to values converted to $X, Y, Z$ space)

Analysis of laboratories in X, Y, Z space, adjusted data, samples C91-C92

Annalysis of laboratories in X, Y, Z space, adjusted data, samples C93-C94.

Analysis of laboratories in L, a, b space, adjusted data, samples C91-C92

Analys is of laboratories in L, a, b space, adjusted data, samples C93-C94

Reflectance at 40 wavelengths, $45 / 0$ Reflectance factor, C92, C94, W14

Spectrophotometric curves of samples C91, C92, C93, C94, W14 
Specimens of a white sample were distributed to the participants along with the usual two pairs of colored specimens, and each participant was asked to return measurement data for the white specimen, reporting results in the same manner as for the colored specimens.

As a first step, three laboratories were selected to serve as "reference" laboratories for the purposes of this analysis and the average of their $X, Y, Z$ values for the white sample were computed. Next, the ratios of the participants data to the combined reference laboratory values were calculated for each participant (transformed to $X, Y, Z$ space if necessary). These ratios are shown in the White Sample Analysis tables.

Two observations can be made about the data in the White Sample Analys is tables. First, the participants as a whole tend to be high compared with the combined average values obtained by the selected reference laboratories. Second, a few participants had noticeably extreme values for one or more of the components and these participants especially should look to the cause.

Next, the ratios in the White Sample Analysis tables were used to "adjust" the data of the normal data tables to obtain the adjusted data table values. The adjustment consisted of dividing the $X, Y, Z$ values of the normal data tables by the respective ratios in the White Sample Analysis tables.

The significant change in the adjusted data tables is in the SD OF MEANS. Comparison of these among-laboratory standard deviations with those in the normal data tables, shows considerable reduction for $X, Y, Z$. Thus part, at least, of the disagreement among participants is due to errors in standardization that could be corrected through use of an agreed-upon white standard. There is no similar significant change for $\Delta X, \Delta Y$, and $\Delta Z$. 


\section{$\triangle E$ Calculation}

$\Delta E$ is calculated in the Color and Color Difference Collaborative Reference Program by the FMC2* equations as follows:

The yellow-blue chromatic difference is

$$
\Delta C_{1}=K_{1} S(P \Delta P+Q \Delta Q) / b D^{2}-K_{1} \Delta S / b \text {; }
$$

the lightness difference is

$$
\Delta L=0.279 K_{2}(P \Delta P+Q \Delta Q) / a D ;
$$

and the red-green chromatic difference is

$$
\Delta C_{3}=K_{1}(Q \Delta P-P \Delta Q) / a D \text {. }
$$

The quantity, D, is an abbreviation,

$$
\begin{aligned}
& D=\left(P^{2}+Q^{2}\right)^{\frac{1}{2}} \cdot \\
& K_{1}=0.55669+0.049434 Y-0.82575 \cdot 10^{-3} \gamma^{2}+ \\
& \quad 0.79172 \cdot 10^{-5} \gamma^{3}-0.30087 \cdot 10^{-7} \gamma^{4}, \\
& K_{2}=0.17548+0.027556 \gamma-0.57262 \cdot 10^{-3} \gamma^{2}+ \\
& \quad 0.63893 \cdot 10^{-5} \gamma^{3}-0.26731 \cdot 10^{-7} Y^{4}, \\
& a^{2}=17.3 \cdot 10^{-6}\left(P^{2}+Q^{2}\right) /\left[1+2.73 P^{2} Q^{2} /\left(P^{4}+Q^{4}\right)\right], \\
& b^{2}=3.098 \cdot 10^{-4}\left(S^{2}+0.2015 Y^{2}\right) \\
& P=0.724 X+0.382 Y-0.098 Z, \\
& Q=-0.48 X+1.37 Y+0.1276 z, \\
& S=0.686 Z, \\
& \Delta E=\left[(\Delta C)^{2}+(\Delta L)^{2}+\left(\Delta C_{3}\right)^{2}\right]^{\frac{1}{2}}
\end{aligned}
$$

*Friele-MacAdam-Chickering metric 
XYZ ANALYSIS

C244

C407

C414

C416A

C416B

C422

C453

C476

C479A

C479B

C511

C521B

C522

C528

C534

C545

C548

C613

C627

C631A

C631B

C632

C638

C639

C644

C545

C657

C660

C661

C671A

C671B

C672

C675

C230

C475

C517

C567

C600

C648

C655

C677 not specified

RIE-LEE

Macbeth MS2000

Diano Hardy II Poly

Diano Hardy Monochromatic II

Gardner SL-31

Hunterlab D54

not specified

$\mathrm{AC}-3$

not specified

Diano Match-Scan

Xerox

Color Guard Sensor W/Gardner PG 5500

Hunter D54P5

Macbeth MS2000

Diano-Hardy Automate

Gardner SL-20

Macbeth MS2000

Zeiss RFL3

ACS Spectra-Sensor

ACS 500 Spectra-Sensor

ACS Spectra-Sensor

Gardner SL-20

Diano Match-Scan

Macbeth MS2000

ACS Spectra-Sensor

ACS Spectra-Sensor

ACS $500 \mathrm{w} / \mathrm{Spectra}$ Sensor

not specified

Diano Match-Scan

Diano Match-Scan

Gardner XL-20

ACS Spectra-Sensor

\section{Lab ANALYSIS}

Hunterlab D25-3

Hunterlab D25-A, nonstandard conditions

Hunterlab D2502

Hunterlab D25

Gardner SL-20

not specified

Hunterlab D54 P-5

Hunterlab D38 


\section{NOTES ON NBS REFLECTANCE VALUES}

The tables on page 17 of this report contain absolute reflectance values measured at 40 wavelengths $(380-770 \mathrm{~nm})$ and tristimulus values for three of the five samples covered by this report. The measurements were performed by the Radiometric Physics Division of the National Bureau of Standards.

These values represent state-of-the-art color measurements on a single specimen of each sample. Thus the results give an accurate picture of the values for single specimens and are not an accurate measure of the whole sample population. Participants should be aware of this concept when comparing their measurements to these NBS values. 
MEAN

GRAND MEAN -

(GR. MEAN)

SD OF MEANS -

INST CODE -

F -

M -

$X-$

* -

0 -
The average of individual test determinations.

The average of the individual laboratory MEANS, excluding laboratories flagged (see column F) with an $X$, \#, or + .

The standard deviation of the laboratory MEANS about the GRAND MEAN: an index of the amonglaboratory precision.

Code for instrument type and color space used to report measurements, see first table.

Flag, is based on $\triangle E$ Column with following meaning: Excluded because data were not understood; because of a non-coded variation reported by the laboratory or data received late.

Excluded because data for one sample are missing Excluded from all calculations because $\Delta E$ is beyond (3) standard deviation units.

Included in grand means but results are between two and three standard deviation units. The participant should take this as a warning to reexamine his testing procedure. Included in grand mean analysis.

Note: In addition to flag ( $F$ ) based on delta $E$ column it is also possible to have either a $X$ or an * on individual MEANS as follows:

$X$ - following a MEAN signifies that the mean is greater than 3 SD of MEANS from the GRAND MEAN. The values for this laboratory have been omitted in the calculations invoiving the MEAN for the column.

* - following any of the MEANS signifies that that quantity is greater than 2 but less than 3 of the appropriate standard deviations from the corresponding average. The participant should take this as a warning to reexamine his testing procedures.

$\Delta E$ - Total color difference between two samples. In $X, Y, Z$ analysis it is calculated in MacAdams (FMC II) units. For L, $a, b$ analysis it is calculated in Hunter units. 

INSTRUMENT I DBNTIFICATIEN

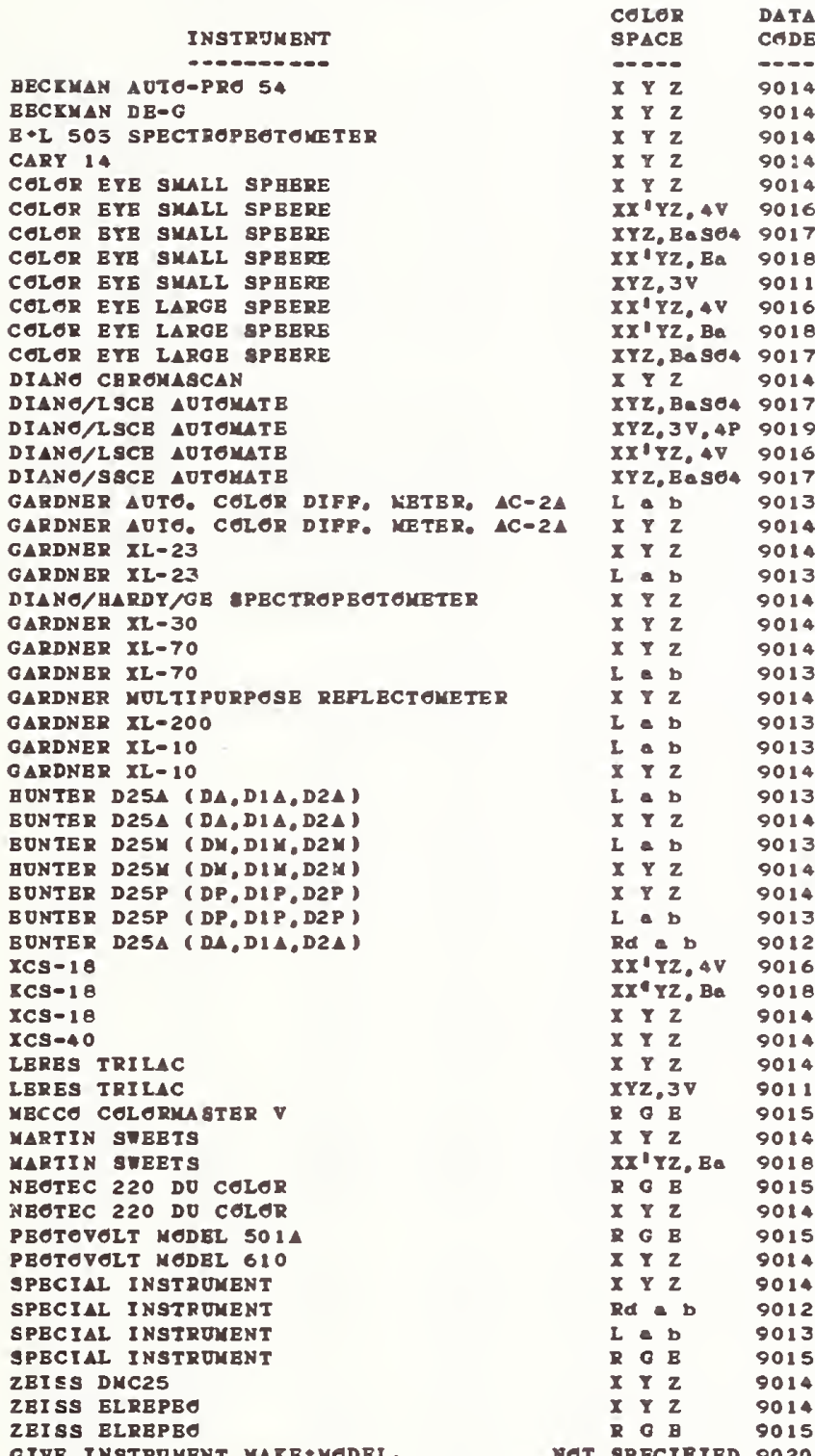

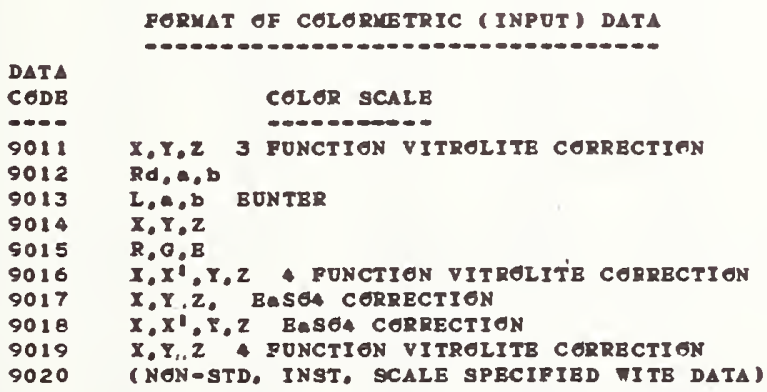



LAB
CEDE $F$ NEAN $X$ SAMPLE $C 9$

\begin{tabular}{|c|c|c|c|c|c|c|}
\hline C162 & $\theta$ & 14.54. & $13.06=$ & 6.56 & $14.62 \%$ & $12.98=$ \\
\hline$C 232$ & $\sigma$ & $17.31 x$ & $15.75 x$ & $7.97 x$ & $17.33 x$ & $15.65 x$ \\
\hline$C 244$ & $\mathbf{x}$ & 13.55 & 12.17 & 5.96 & 13.61 & 12.00 \\
\hline C250 & $\theta$ & $14.68=$ & 13.00 & 6.73 & $14.66=$ & 12.80 \\
\hline C25 I & $\theta$ & 13.74 & 11.80 & 5.91 & 13.91 & 11.90 \\
\hline C278A & $\sigma$ & 13.70 & 12.03 & 5.94 & 13.97 & 12.27 \\
\hline $\mathrm{C} 27 \mathrm{BB}$ & $\sigma$ & 13.60 & $12 \cdot 25$ & 6.25 & 13.80 & $12 \cdot 35$ \\
\hline C314 & $\theta$ & 13.43 & $12 \cdot 12$ & 6.19 & 13.49 & 11.99 \\
\hline$c 372$ & $x$ & 14.11 & 12.70 & $7.39 x$ & 14.28 & 12.80 \\
\hline C407 & $\boldsymbol{\theta}$ & 13.46 & 11.84 & 5.96 & $13 \cdot 48$ & 11.73 \\
\hline C412 & $\theta$ & 14.29 & $12 \cdot 81$ & 6.67 & 14.23 & 12.60 \\
\hline C414 & $\theta$ & 13.74 & $12 \cdot 23$ & 6.11 & 13.76 & 12.10 \\
\hline C416A & $\boldsymbol{\sigma}$ & 13.42 & 11.86 & 5.52 & 13.49 & 11.79 \\
\hline $\mathrm{C} 416 \mathrm{~B}$ & $\theta$ & 13.42 & 11.86 & 5.49 & 13.47 & 11.78 \\
\hline$C 418$ & $\sigma$ & 13.90 & 12.59 & 6.56 & 13.86 & 12.40 \\
\hline C422 & $\theta$ & 13.56 & 11.96 & 6.03 & 13.48 & 11.73 \\
\hline$C 424$ & $\theta$ & 14.27 & 12.76 & 6.69 & 14.25 & 12.59 \\
\hline $\mathrm{CA} 2 \mathrm{~B}$ & $\theta$ & 14.00 & 12.46 & 6.34 & $13 \cdot 96$ & $12 \cdot 28$ \\
\hline C4 37 & $\theta$ & 14.34 & 12.94 & 6.49 & 14.42 & 12.87 \\
\hline$C_{443}$ & $\theta$ & $16.67 \times$ & 12.57 & $5.29=$ & $16.72 x$ & 12.47 \\
\hline $\mathrm{C4} 44$ & 6 & 13.86 & $12 \cdot 41$ & 6.31 & 14.06 & 12.46 \\
\hline$c 445$ & $\boldsymbol{\theta}$ & 13.98 & 12.48 & 6.71 & 14.16 & 12.51 \\
\hline$C 446 A$ & A & 13.81 & 12,27 & 6.23 & 14.12 & 12.46 \\
\hline CA53 & $\theta$ & 13.92 & 12.47 & 6.31 & 13.91 & 12.31 \\
\hline C455 & 6 & 13.79 & 12.31 & 6.28 & 14.07 & 12.44 \\
\hline C459 & $\theta$ & 13.81 & $12 \cdot 33$ & 6.43 & 13.84 & $12 \cdot 21$ \\
\hline C460 & $A$ & 13.72 & 12.27 & 6.22 & 14.02 & 12.42 \\
\hline C462A & $\theta$ & 13.80 & $12 \cdot 20$ & 6.05 & 13.90 & 12.15 \\
\hline $\mathrm{C} 463$ & $\boldsymbol{\theta}$ & 13.87 & $12 \cdot 39$ & 6.33 & 14.25 & 12.62 \\
\hline C467A & A & 13.84 & $12 \cdot 31$ & 6.64 & 14.14 & 12.48 \\
\hline CA67B & 6 & 13.59 & 12.03 & 5.96 & 13.76 & 12.05 \\
\hline C469 & $\theta$ & 13.84 & 12.53 & 5.79 & 13. 88 & $12 \cdot 43$ \\
\hline $\cos 70$ & $\sigma$ & 13.88 & 12.48 & 6.19 & 13.96 & 12.42 \\
\hline$C 472$ & $\sigma$ & 13.72 & 12.29 & 5,73 & 13.75 & 12.19 \\
\hline$C 473$ & 6 & 13.76 & $12 \cdot 48$ & 5.87 & 13.92 & 12.50 \\
\hline C4 76 & $\theta$ & 13.52 & 12.00 & 6.54 & 13.74 & 12.08 \\
\hline C479A & $\theta$ & 13.85 & 12.35 & 6.65 & 13.75 & $12 \cdot 30$ \\
\hline C479B & $A$ & 14.35 & 12.83 & 6.85 & $14.66=$ & $12.99=$ \\
\hline$C 4 B O$ & $\sigma$ & 13.95 & 12.48 & 6.48 & 14.16 & $12 \cdot 55$ \\
\hline$C A B 1$ & $\theta$ & 14.05 & 12.58 & 6.44 & 14.02 & $12 \cdot 43$ \\
\hline $\mathrm{CAB3}$ & $x$ & 14.11 & 12.30 & 6.61 & 14.05 & 12.00 \\
\hline$C 4964$ & 6 & 13.82 & 12.31 & 6.30 & 14.04 & 12.39 \\
\hline CA99C & $\theta$ & 14.00 & $12 \cdot 34$ & 6.49 & 14.49 & 12.71 \\
\hline C503 & $\theta$ & 13.78 & 12.33 & 6.36 & 14.09 & 12.49 \\
\hline C5OB & $\theta$ & 13.65 & 12.12 & 6.18 & 13.70 & 12.03 \\
\hline C5 11 & $\sigma$ & 13.94 & 12.46 & 6.42 & 13.87 & $12 \cdot 24$ \\
\hline $\operatorname{cs} 21 A$ & $\sigma$ & 14.02 & 12.46 & 6.32 & 13. 98 & 12.27 \\
\hline C52 $1 \mathrm{~B}$ & $\boldsymbol{\theta}$ & 13.54 & 11.99 & 6.40 & 13.14 & $11.50=$ \\
\hline C522 & $\theta$ & $14.49=$ & $13.25=$ & $6.98=$ & 14.59 & 13.23 * \\
\hline C524 & A & 14.10 & 12.62 & 6.47 & 14.16 & 12.53 \\
\hline C52 B & $\boldsymbol{\sigma}$ & 13.99 & 12.49 & 6.36 & $14 \cdot 23$ & 12.59 \\
\hline C531 & $\theta$ & 14.07 & 12.61 & 6.54 & 14.02 & 12.40 \\
\hline C532 & $\theta$ & 14.09 & 12.58 & 6.46 & 14.09 & 12.44 \\
\hline C534 & $\sigma$ & 13.56 & 12.10 & 6.03 & $13 \cdot 82$ & 12.21 \\
\hline C536 & $\theta$ & 14.32 & 12.82 & 6.77 & 14.27 & 12.64 \\
\hline C540 & $\sigma$ & $14.61=$ & $13.12=$ & $7.09=$ & $14.60=$ & $12.98 *$ \\
\hline C545 & $\theta$ & $12.99 *$ & $11.56=$ & 5.70 & $13.10 \%$ & $11.54 \mathrm{t}$ \\
\hline C548 & $\theta$ & 13.31 & $11.55=$ & 5.89 & $13.10=$ & $11.25 x$ \\
\hline C549 & $\sigma$ & 13.39 & 11.85 & 5.74 & 13.54 & $11 \cdot 86$ \\
\hline C552 & $\sigma$ & 13.55 & 12.08 & 6.13 & 13.75 & 12.13 \\
\hline C6 12 & d & 13.66 & 12.16 & 6.23 & 13.69 & $12 \cdot 05$ \\
\hline $\cos 13$ & $\sigma$ & 13.92 & $12 \cdot 47$ & 6.12 & 1.01 & $12 \cdot 42$ \\
\hline 0627 & $\theta$ & 13.58 & 12.15 & 5.71 & 13.68 & 12.12 \\
\hline C629 & $\theta$ & 13.74 & $12 \cdot 22$ & 6.22 & 13.74 & $12 \cdot 09$ \\
\hline 0630 & $\sigma$ & 13.83 & 12.34 & 6.33 & $1 \$ .02$ & 12.42 \\
\hline
\end{tabular}

DIPPERENCE C 92 - 991 $\Delta \mathrm{Y} \quad \Delta \mathrm{Z}$
INST

$\triangle E$ CODE LAB

\begin{tabular}{|c|c|c|c|c|c|c|c|c|}
\hline 6.37 & .08 & -.08 & & -19 & & 3.47 & $70 \mathrm{DC}$ & C162 \\
\hline $7.91 x$ & .02 & -.10 & & -.06 & & 2.00 & $70 \mathrm{CK}$ & C232 \\
\hline 5.76 & .07 & -.17 & & -.20 & & $5.05 x$ & $70 \Omega$ & C244 \\
\hline 6.61 & -.02 & -.20 & & -.12 & & 3.55 & $70 Z F$ & C 250 \\
\hline 6.02 & .17 & .10 & & .11 & & $.890^{\circ}$ & $70 Z \mathrm{ZB}$ & C251 \\
\hline 6.08 & .27 & .25 & - & .14 & & $.91 *$ & $70 \mathrm{BL}$ & C278A \\
\hline 6.25 & .20 & .10 & & .00 & & 1.82 & $70 \mathrm{BL}$ & C $278 \mathrm{~B}$ \\
\hline 6. 04 & .05 & $\because 13$ & & -.15 & & 3.79 & $70 \mathrm{CE}$ & C314 \\
\hline 6.38 & .18 & .10 & & -1.01 & $x$ & $7.56 x$ & 7028 & C372 \\
\hline 5.93 & .02 & -011 & & -.03 & & 2.49 & $70 S \Delta$ & C407 \\
\hline 6.46 & .06 & -.21 & & -.22 & & 3.53 & $70 \mathrm{~GB}$ & $C 412$ \\
\hline 5.96 & .01 & .013 & & -15 & & 3.17 & $70 S A$ & CA14 \\
\hline 5.46 & .07 & -.07 & & -.06 & & 2.82 & $70 S A$ & CA16A \\
\hline 5.43 & .05 & -.09 & & -.06 & & 2.77 & $70 S \Delta$ & $\mathrm{C} 416 \mathrm{~B}$ \\
\hline 6.28 & .04 & .019 & & -.28 & & 3.71 & $70 \mathrm{CB}$ & $\cos 18$ \\
\hline 5.75 & -.08 & -.23 & & -.28 & & 3.84 & 70 SA & C422 \\
\hline 6.53 & -.02 & -.16 & & -.16 & & 3.17 & $70 \mathrm{CA}$ & CA24 \\
\hline 6.18 & -.03 & -.18 & & -.16 & & 3.19 & $70 \mathrm{Bg}$ & $C 42 B$ \\
\hline 6.39 & .07 & -.08 & & -.10 & & 2.90 & $70 \mathrm{CB}$ & $\mathrm{C} 437$ \\
\hline $5.17=$ & .05 & -.10 & & -.12 & & 2.99 & $70 \mathrm{EP}$ & C443 \\
\hline 6.30 & .20 & .05 & & .001 & & 2.65 & $700 E$ & $\mathrm{C} 44$ \\
\hline 6.67 & .17 & .02 & & -.04 & & 2.76 & $70 \mathrm{LS}$ & $C 445$ \\
\hline 6.51 & .31 & .19 & & .27 & & 1.76 & $70 \mathrm{GE}$ & $C 446 A$ \\
\hline 6.07 & -.01 & -.16 & & -.23 & & 3.61 & $70 S A$ & $\mathrm{C} 453$ \\
\hline 6.34 & .29 & .13 & & .06 & & 2.49 & $70 \mathrm{KS}$ & C455 \\
\hline 6.32 & .03 & .011 & &. .11 & & 2.92 & $70 G E$ & C459 \\
\hline 6.45 & .29 & .15 & & .23 & & 1.91 & $70 G E$ & $C 460$ \\
\hline 6.05 & .10 & $\because 05$ & & .00 & & 2.63 & $70 \mathrm{HB}$ & C462A \\
\hline 6.62 & .38 & .23 & - & .29 & - & 2.09 & 7020 & C463 \\
\hline 6.93 & .30 & .17 & & .29 & - & 1.76 & 70GE & $C 467 A$ \\
\hline 6.02 & .17 & .02 & & .06 & & 2.35 & $70 \mathrm{HN}$ & $C 467 B$ \\
\hline 5.71 & .04 & -.10 & & .008 & & 2.74 & $700 E$ & C469 \\
\hline 6.10 & .08 & -.06 & & -.09 & & 2.78 & $70 G E$ & C470 \\
\hline 5.76 & .03 & -.10 & & .03 & & 2.25 & 7020 & $c 472$ \\
\hline 5.93 & .17 & .02 & & .06 & & 2.32 & $700 E$ & $c 473$ \\
\hline 6.72 & .22 & .07 & & .19 & & 2.07 & $70 s A$ & $C_{4} 76$ \\
\hline 6.45 & $\because 10$ & -.05 & & -.20 & & $1.00=$ & $70 \Omega$ & C479A \\
\hline $7.00=$ & .31 & .15 & & .15 & & 2.21 & 7081 & $C 479 B$ \\
\hline 6.59 & .21 & .07 & & .11 & & 2.10 & $70 \mathrm{RB}$ & $C 480$ \\
\hline 6.41 & -.03 &. .15 & & -.02 & & 2.25 & $70 \mathrm{ss}$ & $C 481$ \\
\hline 6.26 & -.06 & -30 & & -.35 & & $5.75 x$ & $70 \mathrm{ZF}$ & $\mathrm{C} 483$ \\
\hline 6.37 & .22 & .08 & & .07 & & 2.20 & $70 \mathrm{GE}$ & C496A \\
\hline $7.00=$ & .50 & .37 & $\mathbf{x}$ & .51 & $\pi$ & 2.26 & $70 \mathrm{BL}$ & C499C \\
\hline 6.59 & .31 & .16 & & .23 & & 1.98 & $70 \mathrm{~GB}$ & c503 \\
\hline 6.08 & .04 & -.10 & & -.09 & & 2.85 & $70 \mathrm{GE}$ & $\mathrm{CSOB}$ \\
\hline 6.13 & -.07 & -.22 & & -.29 & & 3.87 & 70SA & C511 \\
\hline 6.19 & -.04 & -.19 & & -.13 & & 3.17 & $70 \mathrm{CA}$ & $\operatorname{c5} 21 \mathrm{~A}$ \\
\hline 5.85 & -.40 & 0.49 & $\mathrm{x}$ & -.56 & $x$ & $4.45=$ & 70 SA & C5218 \\
\hline 6.76 & .10 & -02 & & -.22 & & 3.08 & $705 A$ & C522 \\
\hline 6.38 & .06 &. .09 & & -.10 & & 2.87 & $70 G E$ & $\operatorname{cs}_{24}$ \\
\hline 6.59 & .25 & .10 & & .23 & & 1.92 & 70 SA & C528 \\
\hline 6.25 & -.05 & -.20 & & -.29 & & 3.91 & $70 G E$ & $\operatorname{cs31}$ \\
\hline 6.32 & -.00 & -.14 & & -.14 & & 3.01 & $70 \mathrm{GE}$ & C532 \\
\hline 6.15 & .26 & .11 & & .12 & & 2.34 & $70 \$ A$ & C534 \\
\hline 6.54 & -.05 &. .19 & & -.23 & & 3.34 & $70 \mathrm{KS}$ & C536 \\
\hline $7.02=$ & -.01 &. .14 & & -.07 & & 2.49 & $70 \mathrm{GE}$ & C54O \\
\hline 5.71 & .11 & -.02 & & .01 & & $2 \cdot 32$ & $70.9 A$ & C545 \\
\hline 5.75 & $\because 22$ & -.30 & . & -.14 & & 2.45 & $70 S B$ & $\operatorname{C54} B$ \\
\hline 5.93 & .14 & .01 & & .19 & & 1.94 & $700 \mathrm{E}$ & $\operatorname{c549}$ \\
\hline 6.13 & .20 & .05 & & .00 & & 2.54 & $70 \mathrm{BN}$ & C552 \\
\hline 6.13 & .03 & -.11 & & $\because 10$ & & 2.87 & $70 G B$ & C612 \\
\hline 6.10 & .09 & -.04 & & -.02 & & 2.42 & $703 \Delta$ & C613 \\
\hline 5.69 & .10 & -.03 & & -.02 & & 2.34 & $70 \$ 4$ & C627 \\
\hline 6.10 & .00 & -13 & & -13 & & 2.89 & $70 \mathrm{EN}$ & $\cos 29$ \\
\hline 6.58 & - 19 & .08 & & .25 & & 1.60 & $70 \mathrm{KS}$ & C630 \\
\hline
\end{tabular}




\begin{tabular}{|c|c|c|c|c|c|c|c|c|c|c|c|c|c|}
\hline \multicolumn{2}{|l|}{ LAB } & \multicolumn{3}{|c|}{ SAMPLB C91 } & \multicolumn{3}{|c|}{ SAMPLB C92 } & \multicolumn{3}{|c|}{ DIPFBRBNCE C92 - $\mathrm{CP}_{1}$} & \multicolumn{3}{|c|}{ INST } \\
\hline CODB & $\mathbf{F}$ & MBAN $x$ & MEAN T & MBAN 2 & VBAM $X$ & MBAN & MBAN $z$ & $\Delta x$ & $\Delta \mathbf{Y}$ & $\Delta \mathbf{z}$ & $\Delta B$ & $\mathrm{CODB}$ & $L \Delta B$ \\
\hline C631A & $\boldsymbol{\sigma}$ & $13 \cdot 41$ & 12.17 & 5.74 & 13.64 & 12.25 & 5.83 & . 23 & .08 & .08 & 2. 39 & 70 SA & C63iA \\
\hline C631B & $\sigma$ & 14.11 & 12.64 & 6.44 & 14.07 & $12 \cdot 46$ & 6.28 & .00 & .018 & .016 & 3.08 & 70 SA & C631D \\
\hline $\cos 32$ & $\sigma$ & 13.85 & 12.38 & 6.26 & 13.97 & $12 \cdot 36$ & 6.19 & .12 & .002 & .06 & 2.69 & 7058 & C632 \\
\hline C634 & s & 13.49 & 12.12 & 6.24 & 13.55 & 12.05 & 6.22 & .06 & .07 & .02 & 2.40 & $70 \mathrm{CE}$ & $\cos 34$ \\
\hline $\cos 38$ & $a$ & 13.50 & 11.80 & 6.00 & 13.60 & 11.80 & 6.20 & .10 & .00 & .20 & 1.57 & 70SA & $c 638$ \\
\hline 1039 & $\sigma$ & 13.78 & 12.33 & 6.28 & 14.07 & 12.48 & 6.53 & .29 & .15 & .25 & 1.84 & TOSA & $c 639$ \\
\hline C644 & 6 & 13.73 & 12.27 & 6.03 & 13.86 & 12.27 & 6.09 & .13 & .00 & .06 & 2.08 & $70 \mathrm{SA}$ & C644 \\
\hline c64s & 6 & 14.01 & 12.53 & 6.36 & 14.04 & 12.42 & 6.22 & .03 & .11 & $\therefore 14$ & 2.99 & $705 A$ & C64s \\
\hline$C 646$ & $\theta$ & 13.68 & 13.33 & 5.68 & 13.64 & $13.49 \pi$ & 5.84 & .16 & .16 & is & $.80=$ & $70 \mathrm{CE}$ & c646 \\
\hline c65 & $\sigma$ & 13.49 & 12.25 & 5.75 & 13.70 & 12.33 & 5.85 & .21 & .08 & .10 & 2.08 & TOSA & $\cos 7$ \\
\hline 0660 & $\sigma$ & 13.62 & 12.43 & 5.84 & 13.71 & 12.38 & 5.77 & .09 & .05 & .006 & 2.79 & TosA & c66o \\
\hline C661 & $\sigma$ & $18.80 x$ & $14.64 x$ & $2.05 x$ & $18.95 x$ & $14.63 x$ & $2.01 x$ & .15 & .01 & -.04 & 2,33 & $70 S A$ & c661 \\
\hline 0664 & ฮ & 13.36 & 11.90 & 6.20 & 13.23 & 11.64 & 5.95 & .13 &. .26 & .25 & 3.39 & $70 \mathrm{KC}$ & C664 \\
\hline C67 IA & 0 & 13.56 & 12.27 & 5.94 & 13.61 & 12.15 & 5.74 & .04 &. .12 & -.19 & 3.72 & $70 \$ A$ & C67 IA \\
\hline $\cos 718$ & $\sigma$ & 13.53 & 12.28 & 5.80 & 13.57 & 12.19 & 5.82 & .04 & .00 &. .06 & 2.61 & $708 A$ & C6718 \\
\hline$\infty 671 \mathrm{c}$ & ฮ & 13.67 & 12.12 & 6.11 & 13.60 & 11.90 & 5.92 &. .07 &. .21 & .19 & 3.43 & $70 \mathrm{GC}$ & c67 1C \\
\hline 06710 & $\boldsymbol{\sigma}$ & 14.26 & 12.17 & $5.23 *$ & 14.44 & 12.20 & $5.28 \%$ & .18 & .03 & .04 & 2.24 & $70 \mathrm{~EB}$ & C671D \\
\hline 1672 & 6 & 13.50 & 12.00 & 6.05 & 13.60 & 12.00 & 6.00 & .10 & .00 & .05 & 1.97 & $708 \mathrm{~A}$ & C672 \\
\hline C675 & 0 & 13.96 & 12.49 & 6.45 & 13.85 & 12.24 & 6.13 & -.12 & -.25 & -.32 & 3.76 & TOSA & C675 \\
\hline
\end{tabular}

ORAND MEANS

$\begin{array}{lllllllllllllll}13.62 & 12.34 & 6.21 & 13.91 & 12.30 & 6.18 & -09 & -.04 & -.03 & 2.61\end{array}$

SD GF MEANS

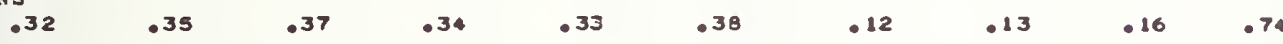

INCLUDED LABS FOR TBIS MBAN 


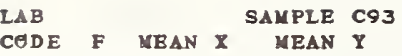

$\begin{array}{lll}C 162 & \theta & 50.66 \\ C 232 & \theta & 57.86 x \\ C 244 & x & 49.13 \\ C 250 & \theta & 50.23 \\ C 251 & \theta & 49.78 \\ & & \\ C 278 A & x & 49.83 \\ C 278 B & \theta & 50.85 \\ C 314 & x & 48.10 \\ C 372 & x & 50.17 \\ C 407 & \theta & 49.79 \\ & & \\ C 412 & \theta & 49.74 \\ C 414 & \theta & 49.35 \\ C 4164 & \theta & 50.07 \\ C 416 B & \theta & 50.08 \\ C 418 & \theta & 48.52 \\ & & \\ C 422 & \theta & 48.76 \\ C 424 & \theta & 49.97 \\ C 428 & \theta & 49.92 \\ C 437 & 9 & 50.63 \\ C 443 & x & 51.50 \\ & & \\ C 444 & \theta & 50.03\end{array}$

C444 50.03

C445 50.20

C446A 49.40

C453 $4 \quad 49.95$

C455 49.76

C459 $4 \quad 49.23$

$\cos 60$ o 49.21

C462A $A \quad 49.90$

c463 50.33

CA67A 50.16

C467B 69.22

C469 60.25

C470 49.74

$442 \bigoplus \quad 49.56$

C473 8.65

C476 49.66

C479A X 49.45

CA79B 51.22

C480 50.17

C481 o 50.75

$\mathrm{C483} \times 49.27$

C496A 49.91

C499C X 51.26

C503 99.73

C508 49.37

C511 19.74

C521A X 50.91

C521B $\times$ 51.54.

C522 49.72

C524 50.33

C528 650.30

C531 50.13

C532 50.26

C534 48.94

C536 50.31

C540 50.11

C545 47.55 .

C548 8.28

C549 49.68

C552 49.48

C612 649.59

C613 650.15

$0627 \quad 6 \quad 4.05$

$\operatorname{C6} 29 \theta 49.43$

C630 49.32 COLAR CELGR DIFFBRBHCB

MEAN $Y$ MEAN $Z$

$\begin{array}{ll}51.58 & 60.11 \\ 58.55 \times & 69.097 \\ 50.07 & 58.31 \\ 51.30 & 60.11 \\ 50.80 & 59.05\end{array}$

51.10

52. 05 .

$48.93 x$

51.20

50.94

50.86

50.43

51.19

51.22

$49.39=$

49.65 .

51.05

50.92

51.47

50.85

51.11

51.28

50.48

51.04

50.93

50.33

50.31

50.90

51.46

51.33

50.23

51.18

50.76

50.56

50.93

50.72

50.35

51.89

51.17

51.67

50.40

51.05

$52.59 \mathrm{X}$

50.76

50.49

50.84

$52.14 \%$

$52.69 x$

51.23

51.43

51.40

51.14

51.36

50.10

51.39

51.23

$48.65 x$

$49.25 x$

50.77

50.47

50.72

51.28

50.85

50.44

50.37

60.35

57.40

59.99

59.13

59.37

58.37

59.62

59.73

58.37

$57.03 *$

59.72

59.48

$61.13=$

$50.51 x$

59.88

60.13

59.00

59.50

59.50

58.64

58.76

59.50

60.18

60.13

58.81

60.63

59.83

59.28

$55.33 x$

59.29

$62.60 x$

60.16

59.88

61.42 .

58.61

59.70

61.14 ㅊ

59.65

59.00

59.33

60.87

61.12.

59.63

60.03

59.97

60.15

60.26

58.04

60.03

59.71

$56.90 x$

57.23 .

59.51

58.99

59.21

59.62

$53.44 x$

58.85

58.99
SAMPLB C94

MBAN $Z$ MBAN $Y$ MEAN $Z$
DIFPERENCE C94-C93

$\Delta \mathrm{I} \quad \Delta \mathrm{Z}$
INST
$60.21 \quad 49.51 \quad 50.70 \quad 58.94$

47.67 .

50.90

50.70

4.9. 34

49.12

49.83

49.83

48.25

48.42

49.74

49.51

50.20

11.47

49.95

48.93

49.69

49.47

48.57

49.16

49.40

49.94

49.98

49.09

49.75

49.45

49.44

48.31

49.43

49.00

49.97

49.72

50.49

48.84

50.00

50.96 .

49.35

49.45

49.38

49.93

$51.79 \pi$

48.94

50.10

50.02

49.85

49.71

48. 88

50.09

50.40

50.15

50.90

50.92

49.11 .

49.27 .

50.76

50.45

50.97

50.51

50.74

50.97

49.96

50.72

50.59

49.61

50.20

50.40

51.01

$51 \cdot 10$

50.04

50.6

50.42

50.41

50.52

50.43

49.95

51.05

50.70

51.36

49.80

51.09

$52.04=$

50.33

50.52

50.44

51.09

52. $89 x$

50.45

51.16

51.15

50.82

50.76

49.99

51.12

49.79

50.85

47.13

49.25

57. $44 \mathrm{I}$

49.84

49.07 


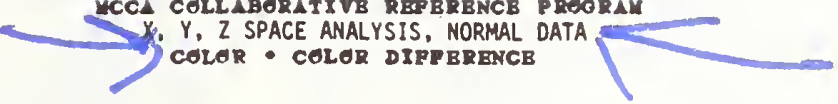

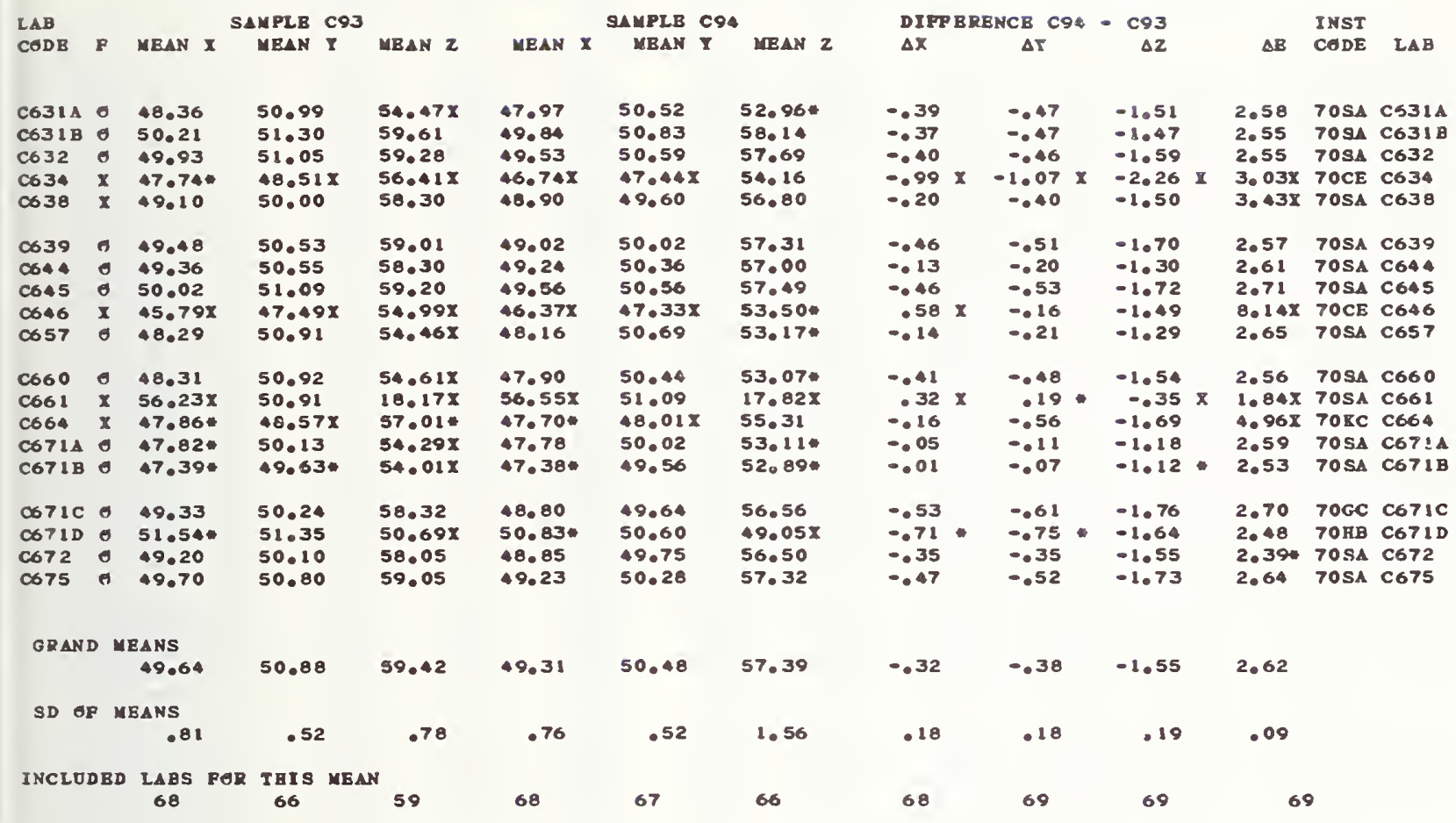



LAB
CODE F MEAN $I$ MAPLE C 1
NEAN A

C105O 34.90

C121 34.80

C122 35.20

C148 835.45

C150 35.25

C152 35.20

C166 35.25

C183 G 35.15

C213 o 34.80

C223 35.22

C230 $6 \quad 35.26$

C2416 35.35

C256 $\times 34.87$

$\begin{array}{lll}c 259 & 6 & 35.15 \\ c 262 & 35.29\end{array}$

C285 35.25

C291 34.95

C317 34.80

C320 30.95

C325 34.93

C340 $\theta 35.45$

C352 35.25

C356 34.80

C380 35.20

C382 35.45

C402 35.11

C417A 35.11

C4178 X 35.02

C420 35.50

C427 34.90

C440 635.40

C442 3 34.80

C454 34.87

C456 35.23

C458 85.07

C475 34.85

C477 35.16

C494 35.70.

C496B $x$
C459A $0.11 x$
35.05

C4998 $\times 35.10$

C506 35.25

$\operatorname{cs} 17$ o 34.95

C538 $36.20 \mathrm{X}$

C542 X 33.96x

$\mathrm{C5} 43 \circ 35.45$

C546A $33.50 X$

C546B 34.50*

C567 35.35

C576 $36.63 x$

C585 6 35.22

C600 34.80

C620 $\theta \quad 35.32$

C633 34.92

$\begin{array}{ll}c 647 & 35.25\end{array}$

C648 o 34.15X

6655 o 34.84

$\cos 62 \quad 35.60$

0674 o 35.40

$\operatorname{cs} 77$ o 35.30

GRAND MEANS

35.14

8.96

13.90

35.05

9.68

13.88

$\begin{array}{lll}8.80 & 14.10 & 34.80 \\ 9.20 & 14.20 & 34.70 \\ 9.30 & 13.95 & 35.10 \\ 9.20 & 14.00 & 35.35 \\ 8.80 & 13.86 & 35.15 \\ 8.80 & 14.00 & 35.05 \\ 8.70 & 13.95 & 35.20 \\ 8.71 & 14.18 & 35.06 \\ 8.60 & 13.20 x & 34.60 \\ 8.66 & 13.84 & 35.13\end{array}$

8. 85

9.10

14.07

13.67

$\begin{array}{ll}0.30 X & 13.70 \\ 8.81 & 13.92\end{array}$

8.70 13.90

8.95

8.46

8.43

8. 90

9.30

8.15 *

8.73

8.78

9.10

9.45

8.80

9.21

8.94

9.41

$.08 x$

8.80

$4.55 x$

8. 70

$0.30 x$

8.75

9.60

8.99

9.15

9.22

$9.70=$

9. 25

9.43

8.64

9.10

14.07

13.80

$9.40 \quad 13.88$
SAMPLE C92

MBAN A MEAN $B$
$9.45 \quad 13.78$

$9.90 \quad 14.20$

13.82

$\begin{array}{ll}1.10 x & 13.80 \\ 9.48 & 13.91\end{array}$

$9.50 \quad 14.10$

9.55

9.80

9.15

9.19

9.05

9.70

10.00

9.65

8.95

9.46

9.91

9.80

10.15

9.52

9.95

9.70

9.94

9.63

10.11

9.70

9.50

9.75

10.00

8.40

$10.90 x$

14.10
13.85
13.70

13.80

13. 82

13. 68

14.10

13. 80

14.00

13. 93

13.99

14.10

13. 85

13.96

13.90

13.77

13.85

13.79

13. $28 x$

13.64

$.14 x$

13.75

13.85

13.80

13.60

$14.30=$

9.57

10.35

9.90

9.76

14.13

1.00

13.70

14.16

9.92

10.50 .

8.78.

9.99

7.70

13.490

9.67

16. $93 x$

$9.41 \quad 14.11$

$9.80 \quad 13.45$

$10.06 \quad 13.76$

(1)

DIFFERENCE C92 - C91

$\begin{array}{rl}9.50 & 14.15 \\ 9.90 & 14.00 \\ 10.00 & 13.90 \\ 9.95 & 14.10 \\ 9.61 & 13.86 \\ & \\ 9.55 & 13.95 \\ 9.55 & 13.85 \\ 9.47 & 14.17 \\ 9.30 & 13.20 x \\ 9.40 & 13.78\end{array}$

35. 32

34.39 .

35.00

35.19

35. 05

35.25

34.85

34.71

35.51

34.95

34.60

35.15

35. 15

35.06

34.55

35.15

35. 28

34.50

34.66

35.15
34.96

35. 02

35.04

34.23

34.95

35.05

35.05

$36.20 x$

$33.55 x$

35.20

$33.40 x$

34.60

35. 30

35.04

34.80

35.62

34.99
35.26

34.19 .

34.63

35.65

35.30

$10.14 \quad 13.59$

$\triangle L \quad \triangle A$

$\triangle B$

.06
$\because 10$
$\because 48$
$\because 15$

.60
.80

- 85

.11

. .20
.30.

.05

.30

.22

.06
-.30

.0
.20

.05

.04
.05
.047
.15
.25

.80

.60

.60
.70

.85
.73

.12

.30

.21

$\because 08$

16

$\because 13$

12

INST
$\triangle E \quad$ CODE LAB

$\begin{array}{rrrrr}-110 & .70 & .05 & .71 & 70 \mathrm{HM} \mathrm{C105} \\ -110 & .70 & -.20 & .73 & 70 \mathrm{HAC} \text { C121 } \\ -110 & .70 & -.05 & .71 & 70 \mathrm{HM} \mathrm{C122} \\ -110 & .75 & .10 & .76 & 70 \mathrm{HA} \mathrm{C148} \\ -10 & .80 & .01 & .81 & 70 \mathrm{HA} \mathrm{C150}\end{array}$

$\begin{array}{llllll}-.15 & .75 & .05 & .77 & 70 \mathrm{AA} & \mathrm{C} 152\end{array}$

$.05 .05 \cdot .10 \quad .86 \quad 7084 \mathrm{C}_{166}$

$.10 \quad .76 \quad-.01 \quad .77 \quad 7084 \mathrm{C}_{183}$

\begin{tabular}{lllll}
-.20 & .70 & .00 & .73 & $70 \mathrm{BM}$ \\
\hline .0213
\end{tabular}

$.06 \quad-60 \quad-.29 .0670 \mathrm{SC} \mathrm{c230}$

$-29$

.05

10

.67
$.8170 \mathrm{SC} C 230$
.982

- $98 \mathrm{~T}$ 7 HX C256

- 82 70RA C250

$.20 \cdot .85 \quad 70 \mathrm{HA} \mathrm{C285}$

$.25 . .7270 \mathrm{TA}$ c291

$.10 \quad .7170 \mathrm{HA} c 317$

.10

- 15

.91. 70सA C320

$.6370 \mathrm{BA}$ C340

. 86 70 EA C352

$.7370 \mathrm{By}$ c.356

.657 она C380

- 86 70HA c382

$.7370 \mathrm{HA} \mathrm{C402}$

- 78 70OE C417A

- 94x 70 en c417B

$.7270 \mathrm{H} \mathrm{C420}$

- 76 70 सA C427 
SAMPLE C93

C105 1051.20

C1216 71.05

C122 1271.15

$\mathrm{Cl} 48$ o 71.45

C150 71.49

C152 6 71.55

C166 971.20

C183 71.46

c213 70.60

$\mathrm{C223} 71.62$

C230 ค 71.17

C241 o 71.20

C256 80.97

C259 71.15

C262 71.69

C285 71.50

c291 671.25

c317 \& 70.90

C320 71.60

C325 71.48

C340 71.62

C352 71.70

c356 71.00

C380 \& 71.65

$\mathrm{C} 382 \theta 71.20$

$\mathrm{C} 402$ ๑ 71.34

C417A 71.46

C417B O 70.93

C420 o 71.55

$\mathrm{C427}$ त 71.30

C440 71.69

$\mathrm{C} 442$ (70.80

$\mathrm{C454} 7 \mathrm{71.63}$

C456 71.33

C45B $\theta 71.16$

C475 71.58

C477 71.59

C494 \& 71.65

C496B X 65.99X

C499A 70.75

C4995 $\theta 71.05$

C506 71.30

C517 071.40

C538 72.10 .

C542 $70.28=$

C543 91.62

C546A 670.40 .

C546B 670.50 .

C567 671.37

C576 $\theta 71.46$

cs8s o 71.29

$0600 \times 70.70$

$\cos 20 \quad 71.68$

$0633 \theta 71.24$

$\operatorname{c647} \sigma 71.74$

C648 o 71.23

C655 81.19

C662 71.90

6674 o 71.50

$0677 \theta 72.53 x$

.031

.14

.30

- 12

.30
.05

.00

. .10
.05

.20
.00

.00
.00

.00

.10

\section{.12}

.22

.00

.16

.00
.20

.01

- 23

- 29

.05

.00
.10

.10

.00

.40

$1.70 x$
$.50=$

.11

.00

.10

.33

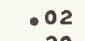

.20
.10

.06

.37

-18
SAMPLB C94

MEAN L MBAN A UGAN B
DIPPBRENCB $\mathrm{C} 94-\mathrm{C} 93$

$\triangle I \quad \Delta A \quad \Delta B$

.10
.00
.30
.600
.031
.000
.05
.34
.10

.20

$\begin{array}{ll}.50 & 71.25 \\ .000 & 71.10\end{array}$

- 57

.60

35

.50
.32

.50

.65

.60

.44

.29

.30

.60

.60

.79
.36

.69

.40

.25

.06
.27

.$: 5$

.65

10

.00
.50

$.50 \quad 71.15$

$.50 \quad 70.95$

$.40 \quad 71.00$

.00 t $\quad 71.70$

.37

.5171 .27

. $30 \quad 70.05 x$

.40

$.45 \quad 71.00$

71.00
71.15

.19

.20

- 27

.40

$-.15$

.00

- 20

.15

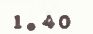

1. 40

1. 30

1. 50

2. 02 ․

1.60
1.40

1.28

1.30

1.34

1. 51

1.60

1. 27

1.50

1.19

1. 50

1.60

1.40

1.33

$\begin{array}{rr}-.08 & 1.16 \\ -.00 \quad 1.25\end{array}$

.00

.20

.10

01

.07
.13

.20

.25

$-25$

- 00

- 28

.13

- 36

- 45

- 15

.00
.00

.20

.10

.65.

$.80 \mathrm{X}$
$.65 \mathrm{.}$

1.25
1.30

1.50

1.60

1. 72

1.20

1.43

1.40

1.00

1. 58

1.30

1.14

1. 03

1.12

1. 55

1. 10

$.01 x$
1.50

1.50

1. 50

1. 40

.08

1. 28

1. 45

1.20

1.30

1.43

1. 53

- $24 \quad 71.10$

$.6 .70 x$

.52

.43

$70.40 \%$

71.28

71.05

71.59

$.22 \quad 011$

.70

70.89

70.80

71.70

71.15
$72.34 x$

\section{. .30}

.045

.010
.05

.17

.10

.10

.10

$-10$

\section{.15}

-.20
-.27

-.27
. .30

.23

.00

.05

.09

.10

.37

.10

.12

-. 38

.15
. .26

- 20

$-14$

10
.03

.90

1.00

1.00

.00
.97

$.10 \div$

$.045 x$

.15
.20

.30

.45
.05

.20

$.30 \mathrm{X}$

1.00

1.00

.95

- 90
- 94

- 94

1.00

- 92

1.00
.88

.32

.10

1.00

.95

-90

- 90

.36

.12

.50

.30

.05

.00

.00 


\begin{tabular}{|c|c|c|c|c|c|c|c|}
\hline \multirow{2}{*}{$\begin{array}{l}\text { LAB } \\
C G D E\end{array}$} & \multicolumn{3}{|c|}{ RATIE $=-(L A B / C E M B I N B D)$} & & PERCENT & PREM C & MBINED \\
\hline & $\mathbf{x}$ & $\mathbf{Y}$ & z & $\operatorname{coD} B$ & $\mathbf{x}$ & $\mathbf{Y}$ & $\mathrm{z}$ \\
\hline C162 & 1.0135 & 1.0107 & 1.0128 & $70 D C$ & 1.35 & 1.07 & 1.28 \\
\hline C232 & 1.1605 & 1.1528 & 1.1517 & $70 \mathrm{cM}$ & 16.05 & $15.2 \theta$ & $15 \cdot 17$ \\
\hline $\mathrm{C} 244$ & .9556 & .5946 & .9942 & $709 x$ &. .44 & -.54 & -.58 \\
\hline C250 & .9964 & .9965 & 1.0003 & $70 \mathrm{ZP}$ & -.36 & -.35 & .03 \\
\hline c251 & 1.0080 & 1.0064 & 1.0059 & $70 \mathrm{ZE}$ & .80 & .64 & .59 \\
\hline$C 278 A$ & 1.0163 & 1.0155 & 1.0377 & $70 B I$ & 1.63 & 1.55 & 3.77 \\
\hline C $278 B$ & 1.0268 & 1.0271 & 1.0236 & $708 L$ & 2.68 & 2.71 & 2.36 \\
\hline $\operatorname{cs} 14$ & .9736 & .9692 & .9711 & $70 \mathrm{CB}$ & -2.64 & -3.08 & -2.89 \\
\hline c 372 &.$\$ \$ 53$ & 1.0075 & 1.0115 & $70 \mathrm{ZB}$ & -.47 & .75 & 1.15 \\
\hline $\mathrm{C} 407$ & 1.0123 & 1.0124 & 1.0105 & $708 A$ & 1.23 & 1.24 & 1.05 \\
\hline $\operatorname{ces} 12$ & 1.0057 & 1.0059 & 1.0065 & $70 \mathrm{~GB}$ & .57 & .59 & .65 \\
\hline $\mathrm{C}_{414}$ & .9902 & .9900 & .9828 & $705 A$ & -.98 & -1.00 & -1.72 \\
\hline C416A & 1.0217 & 1.0214 & 1.0188 & $70 S A$ & 2.17 & 2.14 & 1.88 \\
\hline$C 416 B$ & 1.0241 & 1.0251 & 1.0233 & $70 S A$ & 2.41 & 2.51 & $2 \cdot 33$ \\
\hline$C 418$ & .9835 & .9799 & .9957 & $70 C B$ & -1.65 & -2.01 &. .43 \\
\hline $\mathrm{CA} 22$ & .5906 & .9888 & .9849 & $70 \$ A$ &. .94 & -1.12 & -1.51 \\
\hline $\mathrm{C} 424$ & 1.0086 & 1.0088 & 1.0097 & $70 \mathrm{CA}$ & .86 & .88 & .97 \\
\hline$C 42 B$ & 1.0085 & 1.0066 & 1.0106 & ТОвВ & .85 & .66 & 1.06 \\
\hline C437 & 1.0126 & 1.0089 & 1.0249 & $7 O C B$ & 1.26 & .89 & 2.49 \\
\hline$c 443$ & 1.0442 & 1.0056 & .0528 & $708 B$ & 4.42 & .56 & -14.72 \\
\hline $\mathrm{C} 444$ & 1.0086 & 1.0089 & 1.0125 & $70 G E$ & .86 & .89 & 1.25 \\
\hline C445 & 1.0109 & 1.0101 & 1.0137 & 70Ls & 1.09 & 1.01 & 1.37 \\
\hline$C 446 A$ & 1.0028 & 1.0024 & 1.0043 & $70 \mathrm{~GB}$ & .28 & .24 & .43 \\
\hline C453 & 1.0092 & 1.0085 & 1.0045 & $70 s A$ & .92 & .85 & .45 \\
\hline$c 455$ & 1.0062 & 1.0083 & 1.0025 & $70 \mathrm{rs}$ & .62 & .83 & .25 \\
\hline C459 & .9972 & .9976 & .9957 & $700 \mathrm{~B}$ & -.28 & -.24 & -.43 \\
\hline $\mathrm{C} 460$ & 1.0074 & 1.0085 & 1.0055 & $70 \mathrm{GE}$ & .74 & .85 & .55 \\
\hline C462A & 1.0050 & 1.0036 & 1.0089 & $70 \mathrm{BB}$ & .50 & .36 & .89 \\
\hline C463 & 1.0128 & 1.0117 & 1.0147 & $702 D$ & 1.28 & 1.17 & 1.47 \\
\hline C467A & 1.0188 & 1.0200 & $1.02 \$ 0$ & $70 G E$ & 1.88 & 2.00 & 2.40 \\
\hline$C 467 B$ & 1.0012 & .9997 & 1.0057 & $70 \mathrm{BN}$ & .12 & -.03 & .57 \\
\hline$c 469$ & 1.0175 & 1.0161 & 1.0278 & $700 \mathrm{E}$ & 1.79 & 1.61 & 2.78 \\
\hline$C 470$ & 1.0101 & 1.0093 & 1.0142 & $70 G B$ & 1.01 & .93 & 1.42 \\
\hline $\mathrm{C}_{4} 72$ & 1.0035 & 1.0031 & 1.0058 & $702 \mathrm{D}$ & .35 & .31 & .58 \\
\hline$C 473$ & .9866 & 1.0110 & .9365 & $70 G E$ & -1.34 & 1.10 & -6.35 \\
\hline$C 476$ & 1.0046 & 1.0041 & 1.0039 & $705 \mathrm{~A}$ & .46 & .41 & .39 \\
\hline C479A & .9994 & 1.0009 & .9880 & $709 A$ & -.06 & .09 & -1.20 \\
\hline$C 479 B$ & 1.0117 & 1.0119 & 1.0130 & $709 A$ & 1.17 & 1.19 & 1.30 \\
\hline $\mathrm{C} 480$ & 1.0103 & 1.0081 & 1.0148 & $70 \mathrm{~B}$ & 1.03 & .81 & 1.48 \\
\hline$C 481$ & 1.0189 & 1.0163 & 1.0351 & 7ors & 1.89 & 1.63 & 3.51 \\
\hline$C 483$ & .9942 & .9922 & .9936 & $70 \mathrm{ZF}$ & -.58 &. .78 & -.64 \\
\hline C496A & 1.0174 & 1.0177 & 1.0166 & $700 \mathrm{E}$ & 1.74 & 1.77 & 1.66 \\
\hline $\mathrm{C} 499 \mathrm{C}$ & 1.0277 & 1.0280 & 1.0299 & TOBL & 2.77 & 2.80 & 2.99 \\
\hline C503 & 1.0179 & 1.0181 & 1.0215 & $700 E$ & 1.79 & 1.81 & 2.15 \\
\hline C50s & 1.0067 & 1.0073 & 1.0070 & $700 B$ & .67 & .73 & .70 \\
\hline CS 11 & 1.0033 & 1.0032 & 1.0012 & $70 S A$ & .33 & .32 & .12 \\
\hline C521A & 1.0217 & 1.0227 & 1.0242 & $70 \mathrm{CA}$ & 2.17 & 2.27 & $2 \cdot 42$ \\
\hline $\operatorname{cs} 21 \mathrm{~B}$ & 1.0223 & 1.0226 & 1.0236 & 7051 & 2.23 & 2.26 & $2 \cdot 36$ \\
\hline c5 22 & .5984 & .5976 & .5920 & $70 S A$ &. .16 & -.24 & -80 \\
\hline $\operatorname{cs} 24$ & 1.0184 & 1.0192 & 1.0189 & $700 E$ & 1.84 & 1.92 & 1.89 \\
\hline $\mathrm{C5} 28$ & 1.0112 & 1.0107 & 1.0057 & $70 s \Delta$ & 1.12 & 1.07 & .57 \\
\hline C531 & 1.0185 & 1.0171 & 1.0231 & $70 G B$ & 1.85 & 1.71 & 2.31 \\
\hline c532 & 1.0206 & 1.0212 & 1.0243 & 7OOE & 2.06 & 2.12 & $2 \cdot 43$ \\
\hline C534 & .9814 & .9822 & .9721 & $70 s A$ & -1.86 & -1.78 & -2.79 \\
\hline $\operatorname{c5} 36$ & 1.0110 & 1.0104 & 1.0116 & $70 \mathrm{Rg}$ & 1.10 & 1.04 & 1.16 \\
\hline $\mathrm{c} 540$ & 1.0138 & 1.0135 & 1.0126 & $700 E$ & 1.38 & 1.35 & 1.26 \\
\hline c5 45 & .5703 & .9709 & .9738 & $70 \mathrm{BA}$ & -2.97 & -2.91 & -2.62 \\
\hline C548 & .9857 & .9051 & .9811 & 7058 & -1.43 & -1.49 & -1.89 \\
\hline C5 49 & 1.0092 & 1.0101 & 1.0113 & $700 B$ & .92 & 1.01 & 1.13 \\
\hline$c 552$ & 1.0027 & 1.0008 & 1.0071 & $70 \mathrm{BN}$ & .27 & .08 & .71 \\
\hline c612 & 1.0108 & 1.0118 & 1.0096 & $70 \mathrm{CB}$ & 1.08 & 1.18 & .96 \\
\hline$c 613$ & 1.0077 & 1.0081 & 1.0008 & $708 A$ & .77 & .81 & .08 \\
\hline c627 & .9632 & .9963 & .8956 & $70 s A$ & -3.68 & -.37 & -10.44 \\
\hline C629 & 1.0000 & .9988 & 1.0031 & $70 B N$ & -.00 & -.12 & .31 \\
\hline c630 & 1.0002 & .9995 & 1.0021 & $70 \mathrm{rs}$ & .02 & -.05 & .21 \\
\hline C631A & .9830 & 1.0140 & .9251 & $709 \mathrm{~A}$ & -1.70 & 1.40 & -7.49 \\
\hline C631B & 1.0154 & 1.0145 & 1.0096 & TOSA & 1.54 & 1.45 & .56 \\
\hline C632 & 1.0121 & 1.0006 & 1.0035 & 7054 & 1.21 & .06 & .35 \\
\hline c634 & .9688 & .9642 & .9629 & $70 \mathrm{CB}$ & -3.12 & -3.58 & -3.71 \\
\hline$c 638$ & 1.0011 & .9987 & .9980 & $70 S A$ & .11 & .013 &. .20 \\
\hline C639 & .5574 & .9966 & .9945 & $70 S A$ & -.26 & -.34 &. .55 \\
\hline C644 & .9933 & .9954 & .9852 & $708 \mathrm{~A}$ & -.67 &. .46 & $-1 \cdot 48$ \\
\hline C645 & 1.0104 & 1.0095 & 1.0016 & $70 S A$ & 1.04 & .95 & .16 \\
\hline C646 & .8976 & .9038 & $.89 e 8$ & TOCE & -10.24 & -9.62 & -10.12 \\
\hline c657 & .9811 & 1.0123 & .9244 & 7034 & -1.89 & 1.23 & -7.56 \\
\hline 0660 & .9828 & 1.0132 & .9279 & $709 \mathrm{~A}$ & -1.72 & 1.32 & -7.21 \\
\hline $\cos 61$ & 1.1350 & 1.0126 & 3076 & $705 \mathrm{~A}$ & 13.90 & 1.26 & -69.24 \\
\hline C664 & .9638 & .9565 & .9621 & $70 \mathrm{OrC}$ & -3.62 & -4.35 & -3.79 \\
\hline C671A & .9678 & .9920 & .9176 & $70 S_{A}$ & -3.22 & -.80 & -8.24 \\
\hline C671B & .9598 & .9832 & .9138 & $709 A$ & -4.02 & -1.68 & -8.62 \\
\hline C671C & .9950 & .9942 & .9902 & $700 \mathrm{C}$ & -.50 & -.59 & -.98 \\
\hline C671D & 1.0330 & 1.0094 & .8355 & $70 \mathrm{BB}$ & 3.30 & .94 & $-14 \cdot 45$ \\
\hline c672 & .9983 & .9971 & .9899 & $70 S A$ & -.17 & -.29 & -1.01 \\
\hline C675 & 1.0101 & 100094 & 1.0036 & $708 \mathrm{~A}$ & 1.01 & .94 & .36 \\
\hline
\end{tabular}




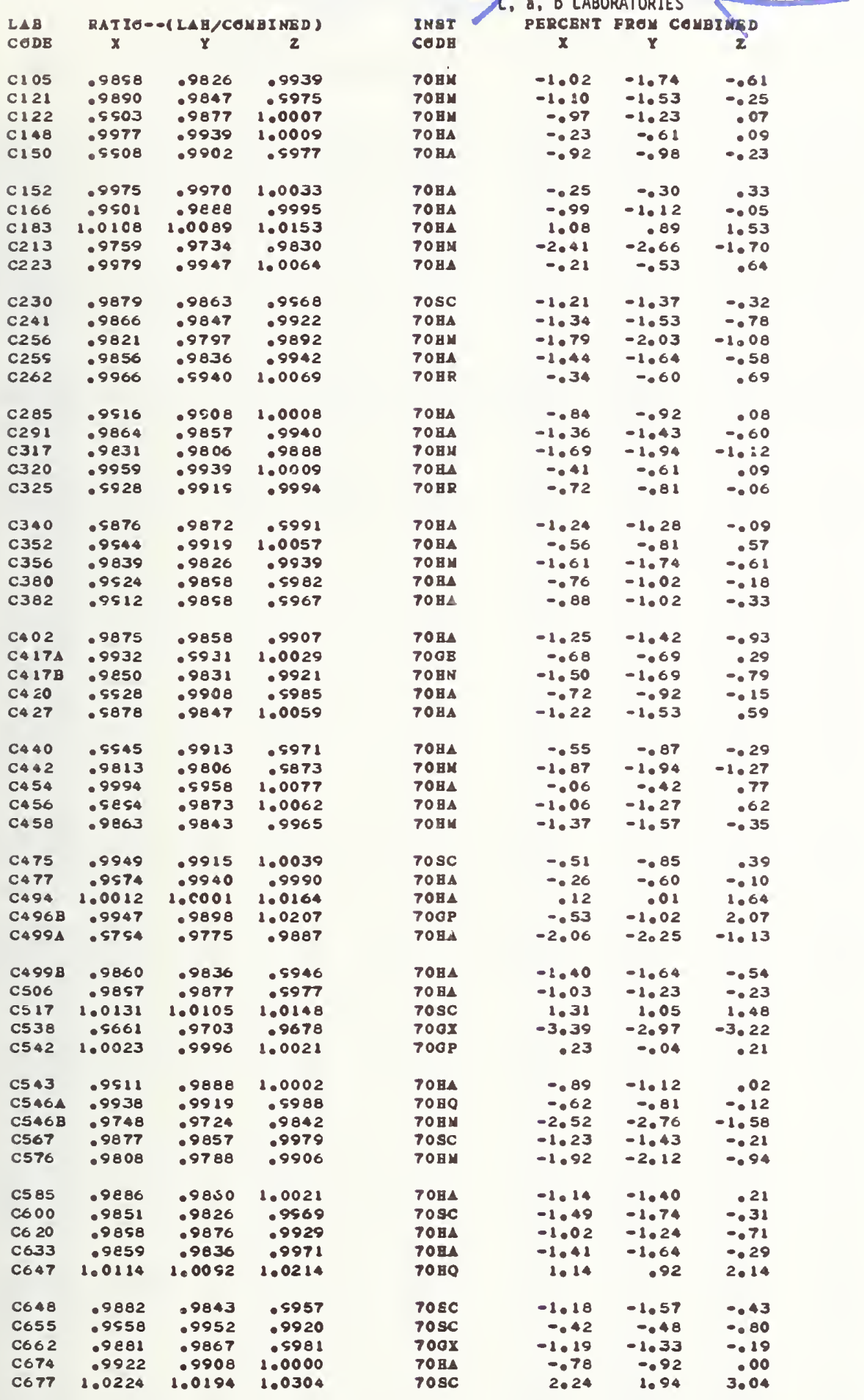




\begin{tabular}{|c|c|c|c|c|c|c|c|c|c|c|c|c|c|c|c|c|}
\hline C162 & $\theta$ & $14.35=$ & 12.93 & 6.48 & 14.43 & $12.85=$ & 6.29 & .08 & & -.08 & & -.19 & & 3.46 & $70 \mathrm{DC}$ & C162 \\
\hline C232 & $d$ & $14.91 x$ & $13.66 x$ & $6.92 \%$ & $14.93 x$ & $13.58 x$ & $6.87=$ & .02 & & .09 & & .05 & & 1.89 & $70 \mathrm{CM}$ & $\mathrm{C} 232$ \\
\hline $\mathrm{C} 244$ & $x$ & 13.61 & 12.24 & 5.99 & 13.68 & 12.06 & 5.79 & .07 & & -.18 & &. .20 & & $5.06 \pi$ & $70 S A$ & C 244 \\
\hline C250 & $\theta$ & $14.73 x$ & 13.05 & 6.73 & 14.71 & 12.84. & 6.61 & .02 & & -.20 & & -.12 & & 3.55 & $70 \mathrm{ZF}$ & C250 \\
\hline C25 1 & $\theta$ & 13.63 & 11.73 & 5.88 & 13.80 & 11.82 & 5.98 & .17 & & .10 & & .11 & & - $89 \%$ & $70 \mathrm{ZE}$ & C251 \\
\hline C278A & $\theta$ & 13.49 & $11 \cdot 85$ & $5.72 \cdot$ & $13 \cdot 75$ & 12.09 & 5.86 & .27 & & .24 & - & .13 & & $.90 *$ & $70 B L$ & C $278 A$ \\
\hline $\mathrm{C} 278 \mathrm{~B}$ & $\theta$ & $13 \cdot 24$ & 11.93 & 6.11 & 13.44 & 12.02 & 6.11 & .19 & & .10 & & .00 & & 1.80 & $70 B L$ & $C 278 B$ \\
\hline $\cos 14$ & $\theta$ & 13.80 & 12.50 & 6.38 & 13.85 & 12.37 & 6.22 & .05 & & -.13 & &. .16 & & 3.83 & $70 \mathrm{CE}$ & C314 \\
\hline C372 & $x$ & 14.18 & 12.61 & $7.31 x$ & 14.35 & 12.71 & 6.30 & .18 & & .10 & & -1.00 & $x$ & $7.51 x$ & $70 \mathrm{ZE}$ & $c 372$ \\
\hline C407 & $\theta$ & $13 \cdot 30$ & 11.70 & 5.90 & $13 \cdot 32$ & $11.59=$ & 5.87 & .02 & &. .11 & & .03 & & 2.48 & $70 S A$ & $C 407$ \\
\hline C412 & e & 14.21 & 12.74 & 6.63 & 14.15 & 12.53 & 6.42 & -.06 & & -.21 & & -.21 & & 3.52 & $70 \mathrm{GE}$ & $C 412$ \\
\hline $\mathrm{CA} 14$ & $\theta$ & 13.88 & 12.35 & 6.22 & 13.90 & 12.22 & 6.06 & .02 & & .013 & & .15 & & 3.19 & 7091 & $C 414$ \\
\hline C416A & $\theta$ & $13.14 \%$ & 11.62 & $5.42 x$ & $13.21 *$ & $11.55=$ & $5.36 x$ & .07 & & -.07 & & .006 & & 2.80 & $70 \mathrm{SA}$ & $C 416 A$ \\
\hline$C 416 B$ & G & 13.104 & $11.57 \%$ & $5.37 x$ & 13.16 & $11.49=$ & $5.31 x$ & .05 & & .08 & & .006 & & 2.74 & $70 \mathrm{sA}$ & $C 416 B$ \\
\hline$C A 18$ & $\theta$ & 14.14 & 12.84 & 6.59 & 14.09 & 12.65 & 6.31 & .04 & & -.19 & & -.28 & & 3.72 & $70 \mathrm{CE}$ & $C 418$ \\
\hline C.22 & $\theta$ & 13.69 & 12.10 & 6.12 & $13 \cdot 61$ & 11.87 & 5.84 & .008 & & -.23 & & -.28 & & 3.86 & $70 S A$ & $c 422$ \\
\hline C424 & $\boldsymbol{\theta}$ & 14.15 & 12.65 & 6.63 & 14.13 & 12.48 & 6.47 & -.02 & &. .16 & &. .16 & & 3.16 & $70 \mathrm{CA}$ & $C 424$ \\
\hline C4 28 & $\theta$ & 13.88 & 12.38 & 6.27 & 13.85 & 12.20 & 6.12 & .003 & &. .18 & &. .16 & & 3.18 & $70 \mathrm{gBB}$ & $C 428$ \\
\hline $\mathrm{C} 437$ & $\theta$ & 14.17 & 12.83 & 6.34 & 14.24 & 12.75 & 6.24 & .07 & & -.08 & & .10 & & 2.88 & $70 \mathrm{CE}$ & $c 437$ \\
\hline $\cos 3$ & $\theta$ & $15.96 x$ & 12.50 & 6.20 & $16.01 x$ & 12.40 & 6.07 & .05 & & -.10 & & 013 & & 3.09 & $70 \mathrm{BB}$ & $C 443$ \\
\hline$C 444$ & $\theta$ & 13.75 & 12.30 & 6.24 & 13.94 & 12.35 & 6.23 & .20 & & .05 & & .001 & & 2.64 & $70 \mathrm{GE}$ & C4.4 \\
\hline C445 & $\theta$ & 13.83 & 12.36 & 6.62 & 14.01 & 12.38 & 6.59 & .17 & & .02 & & -.04 & & 2.74 & 70Is & $C_{445}$ \\
\hline$C 4461$ & $\theta$ & 13.77 & 12.25 & 0.21 & 14.09 & 12.43 & 6.48 & .31 & & .18 & & .27 & & 1.76 & $70 \mathrm{GE}$ & $C 446 A$ \\
\hline C453 & $\theta$ & 13.79 & 12.37 & 6.28 & 13.78 & 12.21 & 6.05 & -.01 & & -.16 & &. .23 & & 3.60 & $70 S A$ & $c 453$ \\
\hline C455 & $\theta$ & 13.71 & 12.21 & 6.26 & 13.99 & 12.34 & 6.33 & .28 & & .13 & & .06 & & 2.49 & $70 \mathrm{xs}$ & $c 455$ \\
\hline C459 & $\theta$ & 13.85 & 12.36 & 6.46 & 13.88 & 12.24 & 6.35 & .03 & & 0.11 & & 011 & & 2.92 & TOGE & $C 459$ \\
\hline $\operatorname{cs} 60$ & $\theta$ & 13.62 & 12.17 & 6.19 & 13.92 & $12 \cdot 32$ & 6.42 & .29 & & .15 & & .23 & & 1.91 & $70 \mathrm{GE}$ & C460 \\
\hline C462A & $\theta$ & 13.73 & 12.16 & 6.00 & $13 \cdot 83$ & 12.11 & 6.00 & .10 & & -.05 & & .00 & & 2.63 & $70 \mathrm{BB}$ & C462A \\
\hline C463 & $\theta$ & 13.69 & 12.25 & 6.24 & 14.07 & 12.47 & 6.52 & .38 & - & .22 & 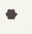 & .29 & $*$ & 2.08 & $70 \mathrm{ZD}$ & $C 463$ \\
\hline$C 467 A$ & 6 & 13.59 & 12.07 & 6.48 & 13.88 & 12.24 & 6.77 & .29 & & .17 & & .28 & $\bullet$ & 1.75 & TOGE & $C 467 A$ \\
\hline C.67B & 6 & 13.57 & 12.03 & 5.93 & 13.74 & 12.06 & 5.99 & .17 & & .03 & & .05 & & 2.35 & $708 \mathrm{~N}$ & $C 467 B$ \\
\hline C469 & e & 13.60 & 12.33 & $5.64 \%$ & 13.64 & 12.24 & 5.56 & .04 & & -.09 & & .08 & & 2.72 & TOGE & $C 469$ \\
\hline$C_{4} 70$ & a & 13.74 & 12.37 & 6.11 & 13.82 & $12 \cdot 31$ & 6.02 & .08 & & -.06 & & -.09 & & 2.77 & TOGE & $C 470$ \\
\hline C 472 & $\theta$ & 13.67 & 12.25 & 5.70 & 13.70 & 12.15 & 5.73 & .03 & & -.10 & & .03 & & 2.25 & $70 \mathrm{ZD}$ & $c 472$ \\
\hline C473 & $\sigma$ & 13.95 & 12.35 & 6.27 & 14.11 & 12.37 & 6.34 & .17 & & .02 & & .07 & & 2.32 & $70 O B$ & $c 473$ \\
\hline$C_{476}$ & 6 & 13.46 & 11.96 & 6.51 & 13.68 & 12.03 & 6.70 & .22 & & .07 & & .18 & & 2.07 & $70 \mathrm{~s} A$ & $C_{4} 76$ \\
\hline $\operatorname{CA79A}$ & $\theta$ & 13.86 & 12.34 & 6.73 & 13.76 & 12.29 & 6.53 & .10 & & -.05 & & -.20 & & $1.01=$ & TOSA & $C 479 A$ \\
\hline C479B & $\theta$ & 14.18 & 12.68 & 6.76 & 14.49. & $12.84 *$ & $6.92 *$ & .31 & & .15 & & .15 & & 2.20 & $70 S A$ & $C 479 B$ \\
\hline$C 480$ & $\theta$ & 13.81 & 12.39 & 6.39 & 14.02 & 12.45 & 6.49 & .21 & & .07 & & .10 & & 2.09 & ТОКв & $C 480$ \\
\hline $\mathrm{CAB} 1$ & $\sigma$ & 13.79 & $12 \cdot 38$ & 6.22 & $13 \cdot 76$ & 12.24 & 6.20 & .03 & & -.15 & & .02 & & 2.23 & $70 \mathrm{ES}$ & $C 4 B_{1}$ \\
\hline C483 & $x$ & 14.19 & 12.40 & 6.66 & 14.14 & 12.09 & 6.30 & .06 & &. .30 & & .36 & & $5.77 x$ & $70 Z F$ & C483 \\
\hline C496A & $\sigma$ & 13.58 & 12.10 & 6.20 & 13.80 & 12.17 & 6.27 & .22 & & .08 & & .07 & & 2.18 & $70 G E$ & $C 496 A$ \\
\hline C499C & $\theta$ & 13.62 & 12.00 & 6.30 & 14.10 & $12 \cdot 36$ & 6.80 & - 48 & $\mathbf{x}$ & .36 & $x$ & .50 & $x$ & 2.23 & $70 \mathrm{BL}$ & $\mathrm{C} 499 \mathrm{C}$ \\
\hline C503 & $\theta$ & 13.54 & 12.11 & 6.23 & 13.84 & 12.27 & 6.45 & .30 & & .16 & & .23 & & 1.97 & $70 \mathrm{GE}$ & c503 \\
\hline C50B & $\sigma$ & 13.56 & 12.04 & 6.14 & 13.61 & 11.94 & 6.04 & .04 & & -.09 & & -.09 & & 2.84 & $700 E$ & C508 \\
\hline C511 & $\theta$ & 13.90 & $12 \cdot 43$ & 6.42 & 13.83 & 12.21 & 6.13 & .007 & & -.22 & & -.29 & & 3.86 & 7084 & C51 I \\
\hline C521A & $a$ & 13.72 & 12.18 & 6.17 & 13.68 & 12.00 & 6.04 & -.04 & & .019 & &. .13 & & 3.14 & $70 \mathrm{CA}$ & C521A \\
\hline C521B & $\theta$ & 13.24 & 11.73 & 6.26 & $12.85 X$ & $11.25 x$ & 5.71 & -.39 & $x$ & -.48 & $x$ &. .54 & $x$ & $4.41 *$ & $70 S A$ & $\mathrm{C521B}$ \\
\hline C522 & $\theta$ & $14.51 \%$ & $13.29 x$ & $7.04=$ & $14.61 \%$ & $13.26 \pi$ & 6.81 & .10 & &. .03 & &. .22 & & 3.09 & $70 S A$ & C522 \\
\hline c524 & $\theta$ & 13.85 & 12.38 & 6.35 & 13.91 & 12.30 & 6.26 & .06 & & -.08 & & .00 & & 2.85 & $700 E$ & C524 \\
\hline C52B & $\theta$ & 13.84 & $12 \cdot 36$ & 6.32 & 14.08 & 12.46 & 6.55 & .24 & & .10 & & .23 & & 1.91 & $70 S A$ & $\operatorname{cs} 28$ \\
\hline C531 & $\theta$ & 13.81 & 12.39 & 6.39 & 13.77 & 12.20 & 6.11 &. .05 & & -.20 & & -.28 & & 3.88 & $700 \mathrm{~B}$ & $\operatorname{cs3} 1$ \\
\hline C532 & $\theta$ & 13.81 & 12.32 & 6.31 & 13.81 & 12.18 & 6.17 & .00 & &. .14 & & -.14 & & 2.98 & $70 \mathrm{GE}$ & c532 \\
\hline C534 & $\theta$ & 13.82 & 12.32 & 6.21 & 14.08 & 12.43 & 6.33 & .26 & & .11 & & .12 & & 2.36 & TOSA & C534 \\
\hline $\operatorname{c5} 36$ & $\sigma$ & 14.16 & 12.69 & 6.70 & 14.12 & 12.51 & 6.47 & .04 & & -118 & & -.23 & & 3.33 & $70 \mathrm{KS}$ & C536 \\
\hline C540 & $\theta$ & 14.42 & 12.95 & $7.01 \%$ & 14.41 & 12.81 & $6.93 *$ & .01 & &. .14 & &. .07 & & 2.48 & $70 \mathrm{CE}$ & C540 \\
\hline C545 & $\theta$ & 13.39 & 11.91 & 5.85 & 13.51 & 11.89 & 5.86 & .11 & & -.02 & & .01 & & 2.34 & $70 S A$ & C545 \\
\hline C54B & $\theta$ & 13.51 & 11.73 & 6.00 & $13.29 *$ & $11.42=$ & 5.86 & -.22 & - & -.30 & - & .014 & & 2.46 & $70 \mathrm{BB}$ & $\operatorname{c5} 48$ \\
\hline C549 & $\sigma$ & $13 \cdot 27$ & $11 \cdot 73$ & 5.68 & 13.42 & 11.75 & 5.87 & .14 & & .01 & & .19 & & 1.94 & $7 O A E$ & C549 \\
\hline C552 & $\sigma$ & 13.52 & 12.08 & 6.09 & 13.71 & 12.13 & 6.09 & .19 & & .05 & & .00 & & 2.53 & $70 \mathrm{BN}$ & C552 \\
\hline $\operatorname{Co} 12$ & $\sigma$ & $13 \cdot 52$ & 12.02 & 6.18 & $13 \cdot 55$ & 11.91 & 6.08 & .03 & &. .11 & & -10 & & 2.86 & $70 G E$ & c612 \\
\hline 0613 & $\theta$ & 13.81 & 12.37 & 6.12 & 13.90 & 12.33 & 6.10 & .09 & & .04 & & .002 & & 2,41 & Tosa & $\cos 13$ \\
\hline 0627 & 0 & 14.10 & 12.20 & 6.38 & 14.20 & 12.16 & 6.36 & .10 & & .004 & & -.02 & & 2.38 & $70 S A$ & C627 \\
\hline 1629 & $\sigma$ & 13.74 & 12.24 & 6.21 & 13.74 & 12.10 & 6.08 & .00 & & -.14 & & -.12 & & 2.89 & $70 \mathrm{BN}$ & 0629 \\
\hline c63o & $\sigma$ & 13.83 & $12 \cdot 35$ & 6.32 & 14.02 & $12 \cdot 43$ & 6.57 & .19 & & .08 & & .25 & & 1.60 & $70 \mathrm{KS}$ & C630 \\
\hline
\end{tabular}

DIFPERENCE CQZ
$\Delta X \quad C Q 1$
$\Delta Y$

DIFPERENCE CQ2 $-C 91$
$\Delta X \quad \Delta Y$

INST

$\triangle E$ CEDE LAB

YAB MEAN 2

MBAN $X$ MEAN $Y$ MEAN $Z$ 

colar - Color DIPPBREHCB

\begin{tabular}{|c|c|c|c|}
\hline LAB & & & AMPLB \\
\hline CODE & $\mathbf{F}$ & $\triangle B A N X$ & MEAN \\
\hline C631A & $\sigma$ & 13.64 & 12.00 \\
\hline $\cos 31 \mathrm{~B}$ & $\bullet$ & 13.90 & $12 \cdot 46$ \\
\hline c632 & 0 & 13.68 & 12.37 \\
\hline C634 & a & 13.93 & 12.57 \\
\hline C638 & $\sigma$ & 13.49 & 11.82 \\
\hline C639 & 6 & 13.82 & $12 \cdot 3 \theta$ \\
\hline C644 & $\sigma$ & 13.83 & 12.33 \\
\hline $\cos 45$ & $\sigma$ & 13.87 & $12 \cdot 41$ \\
\hline C646 & $\theta$ & $15 \cdot 24 x$ & $14.75 x$ \\
\hline c657 & $\theta$ & 13.75 & 12.11 \\
\hline C660 & $\theta$ & 13.86 & 12.27 \\
\hline 1061 & d & $16.51 x$ & $14.46 x$ \\
\hline c66 & o & 13.86 & 12.44 \\
\hline 06711 & $\sigma$ & 14.02 & 12.37 \\
\hline C671B & ฮ & 14.10 & 12.50 \\
\hline $\cos 71 \mathrm{C}$ & $\boldsymbol{\sigma}$ & 13.74 & 12.19 \\
\hline C671D & 0 & 13.81 & 12.06 \\
\hline 0672 & $\sigma$ & 13.52 & 12.04 \\
\hline C675 & o & 13.82 & 12.38 \\
\hline
\end{tabular}
MBAN 2
SAMPLE C92

MBAN $I$ MRAY $Y$ UEAN $Z$
DIFFERENCB $\mathrm{C}_{92}-\mathrm{CO}$

$\Delta \mathbf{X}$

$\Delta \mathbf{Y}$

$\Delta \mathbf{z}$

6.38

$$
6.24
$$

6.49

6.01

13.88

13.86

13.80

12.08
12.28

12.35

$13.99 \quad 12.50$

23. 59

11.82

$6.31 \quad 14.11 \quad 12.53$

6.12

6.35

6.32

13.96

13.90

12.30

13.97

12.18

\subsection{0}

6.22

$6 \cdot 17$

6.46

6.21

.23
.04

.04

112

.06

6.57

6.18

$6 \cdot 21$

6.50

6.33

6.29

13.96

$12 \cdot 22$

16.64x $14.45 x$

6.45

6.47

13.73

14.06

12. 17

12.25

$6.44 \quad 14.14 \quad 12.40$

\subsection{2}

6.54

6.19

6.26

6.37

\subsection{8}

6.12

6.11

13.67

13.98

11.97

12.09

$13.62 \quad 12.08$

13.71

12.13

5. 98

6.17

6.06

6.11

.29
.13

.03

18

.18
.21

.08

.18

.02

.02

.09
-.16

.06

.02

.20

$-15$

.15

.11

.17

.25

.06

$-14$

.17

.11

10
.13

.13
-.13

.05

.05
.01

.26

$-12$

$-.07$

.07
.13

.26

$-21$

.07

.07

.09

$-20$

$-.22$

$-17$

.10

.11

.03

.00

. .25

.05

.05

. .32

$12 \cdot 23$

6.26

.09

.04

.03

2.61

SD GP MEANS

$.27 \cdot 31$

.28

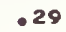

.29

.29

.12

.13

.16

.74

INCLUDBD LABS FOR THIE MBNY

76

79

76

76

79

79

79

79

81 


\begin{tabular}{|c|c|c|c|c|c|c|c|c|c|c|c|c|c|c|}
\hline$I A B$ & & & SUPLE CQ: & & & SAXPLB CQ & & DIFP & $\mathbf{E R}$ & BNCE CQ & - $c 93$ & & INST & \\
\hline$C A D E$ & $\mathbf{P}$ & MEAN $X$ & MEAN $Y$ & MEAN $z$ & MRAN $X$ & MBAN $\mathbf{Y}$ & MEAN $Z$ & $\Delta \mathbf{x}$ & & $\Delta \mathbf{Y}$ & $\Delta \mathbf{z}$ & $\Delta E$ & CEDE & LAB \\
\hline$c 162$ & $\theta$ & 49.99 & 51.04 & 59.36 & 49.54 & 50.53 & 57.68 & -.45 & & -.50 & -1.67 & 2.59 & $7 O D C$ & $c 162$ \\
\hline$C 232$ & $\theta$ & 49.86 & 50.79 & 59.99. & 49.49 & 50.49 & 58.24 &. .36 & & -.30 & -1.74 & 2.51 & $70 \mathrm{CK}$ & $c 232$ \\
\hline$c 244$ & $\mathrm{x}$ & 49.35 & 50.35 & 58.65 & 49.20 & 50.22 & 57.37 & .015 & & .013 & -1.28 & $2.18 x$ & $70 S A$ & $\mathrm{C} 244$ \\
\hline$c 250$ & 6 & $50.42 x$ & 51.48. & 60.09 & $50.02 x$ & 50.98. & 58.44 &. .39 & & .50 & -1.65 & $2.85 \%$ & $702 F$ & $c 250$ \\
\hline C25I & S & 49.38 & 50.48 & 58.70 & 48.68 & 49.68 & 56.84 & .070 & - &. .79 & -1.87 & 2.68 & $702 E$ & C251 \\
\hline C278A & $x$ & 49.03 & 50.33 & $58.02:$ & 48.72 & 49.93 & 56.80 &. .31 & & .40 & $-1 \cdot 22$ & $2 \cdot 11 x$ & $70 \mathrm{BL}$ & C2784 \\
\hline C278B & 0 & 49.52 & 50.68 & 58.96 & 49.18 & 50.24 & 57.44 & .034 & & -.44 & -1.51 & 2.67 & $70 \mathrm{BL}$ & $C 278 B$ \\
\hline $\mathrm{C} 314$ & $x$ & 49.40 & 50.49 & 59.11 & 48.97 & 49.94 & 57.29 & .044 & & -.55 & -1.82 & $3 \cdot 12 x$ & $70 \mathrm{CE}$ & $c 314$ \\
\hline C372 & $\mathrm{x}$ & $50.41 \mathrm{X}$ & 50.82 & 59.31 & $50.08 x$ & 50.52 & 57.79 & -.34 & &. .30 & -1.52 & $2.22 x$ & $70 \mathrm{ZE}$ & $c 372$ \\
\hline C407 & $\theta$ & 49.18 & 50.31 & 58.52 & 49.01 & 50.08 & 57.08 & -.17 & & $-\infty 24$ & -1.44 & 2.76 & $70 S A$ & C407 \\
\hline$C_{4} 12$ & $\theta$ & 45.47 & 50.56 & 58.98 & 49.06 & 50.10 & 57.36 & -.40 & &. .46 & -1.62 & 2.58 & $700 B$ & $\mathrm{C}_{412}$ \\
\hline $\mathrm{C} 414$ & $\theta$ & 49.84 & 50.95 & 59.40 & 49.61 & 50.66 & 57.94 &. .23 & &. .28 & -1.46 & 2.62 & $708 A$ & C 414 \\
\hline$C 416 A$ & $\sigma$ & 45.01 & 50.12 & 58.53 & 48.77 & 49.83 & 57.11 &. .24 & & .29 & -1.41 & 2.50 & $70 S A$ & C4164 \\
\hline$C 416 B$ & $\theta$ & 48.91 & 49.97 & $58 \cdot 37$ & 48.66 & 49.68 & 56.93 & -.24 & &. .29 & -1.44 & 2.55 & $70 S A$ & $C 416 B$ \\
\hline$C 418$ & $\theta$ & 49.34 & 50.40 & 58.63 & 49.06 & 50.12 & 57.14 & -.27 & & -.28 & -1.48 & 2.43 & $70 \mathrm{CE}$ & $c 418$ \\
\hline $\mathrm{C} 422$ & $\sigma$ & 49.23 & 50.22 & 58.51 & 48.88 & 49.83 & 56.91 &. .34 & & -.38 & -1.60 & 2.64 & TOSA & $c 422$ \\
\hline CA 24 & 6 & 49.54 & 50.61 & 59.15 & 49.31 & 50.32 & 57.62 &. .23 & & -.29 & -1.53 & 2.76 & $70 \mathrm{CA}$ & $c 424$ \\
\hline $\cos 28$ & $\sigma$ & 49.50 & 50.59 & 58.85 & 49.10 & 50.12 & 57.20 & -041 & &. .46 & -1.66 & 2.66 & $70 \mathrm{BB}$ & $C 428$ \\
\hline C437 & $\sigma$ & 50.00 . & 51.01 & 59.64 & 49.57 & 50.51 & 58.01 & .043 & & -.50 & -1.63 & 2.62 & $70 \mathrm{CE}$ & $c 437$ \\
\hline $\operatorname{CA4} 3$ & $x$ & 49.32 & 50.57 & 59.23 & 49.29 & 50.23 & 57.72 & .03 & & -.34 & $-1 \cdot 51$ & $4.28 x$ & $70 \mathrm{BB}$ & C 443 \\
\hline CA4 4 & $\theta$ & 49.61 & 50.66 & 59.14 & 49.29 & 50.30 & 57.61 & -.32 & &. .36 & -1.54 & 2.55 & $700 E$ & $\mathrm{C} 444$ \\
\hline CA4 5 & $\theta$ & 49.66 & 50.77 & 59.32 & 49.41 & 50.46 & 57.81 &. .25 & & .30 & -1.51 & 2.68 & 70LS & $c 445$ \\
\hline CA46A & $\theta$ & $\$ 9.27$ & $50 \cdot 36$ & 58.75 & 48.79 & 49.84 & 57.08 & .047 & &. .52 & -1.67 & 2.54 & TOGE & $C 446 A$ \\
\hline C453 & 0 & 49.50 & 50.61 & 59.23 & 49.24 & 50.30 & 57.78 &. .26 & &. .31 & -1.45 & 2.54 & $70 \mathrm{SA}$ & $C \$ 53$ \\
\hline CAs5 & a & 49.46 & 50.52 & 59.35 & 49.17 & 50.17 & 57.81 & .29 & & -.34 & -1.54 & 2.64 & $70 \mathrm{ks}$ & c455 \\
\hline C459 & $\theta$ & 49.37 & 50.45 & 58.89 & 48.71 & 49.73 & 56.99 &. .66 & &. .72 & -1.90 & 2.70 & $70 G E$ & $c 459$ \\
\hline$C 460$ & $\theta$ & $48.85=$ & 49.89 & 58.45 & 48.80 & 49.78 & 57.23 & .05 & & .011 & -1.21 & 2.55 & $70 \mathrm{GE}$ & C460 \\
\hline $\operatorname{cs} 621$ & $\theta$ & 49.65 & 50.72 & 58.97 & 49.16 & 50.22 & 57.29 & .50 & & .50 & -1.68 & $2.34=$ & $70 \mathrm{~EB}$ & C462A \\
\hline$C 463$ & $\theta$ & 49.69 & 50.87 & 59.32 & 49.31 & 50.42 & 57.71 &. .38 & &. .45 & -1.61 & 2.64 & 7OZD & $c 463$ \\
\hline$C 467 A$ & $\theta$ & 49.24 & 50.33 & 58.72 & 49.06 & 50.10 & $57 \cdot 28$ & -.18 & &. .23 & -1.4 & 2.68 & $7 O G E$ & C467A \\
\hline C467B & $\theta$ & 49.16 & 50.25 & 58.48 & 49.03 & 50.06 & 57.09 & -.12 & &. .20 & -1.39 & 2.80 & $70 \mathrm{BN}$ & $C 467 \mathrm{~B}$ \\
\hline C469 & $\theta$ & 49.37 & 50.37 & 58.99 & 48.88 & 49.84 & 57.27 & -.49 & & -.53 & -1.72 & 2.59 & $70 G E$ & $c 469$ \\
\hline$C_{4} 70$ & $\theta$ & 49.24 & 50.29 & 58.99 & 8.96 & 49.96 & $57 \cdot 45$ & -.28 & & -.33 & -1.54 & 2.65 & $70 \mathrm{GE}$ & $c 470$ \\
\hline C472 & $\theta$ & 49.39 & 50.41 & 58.9 & 49.27 & 50.26 & 57.64 & .11 & &. .15 & $-1 \cdot 30$ & 2.50 & $70 \mathrm{ZD}$ & $c 472$ \\
\hline $\cos 73$ & $\theta$ & 49.31 & 50.38 & 59.09 & 48.97 & 49.97 & 57.53 & .34 & &.-40 & -1.56 & 2.61 & $700 E$ & C473 \\
\hline C476 & 6 & 49.43 & 50.52 & 59.06 & 49.20 & 50.23 & 57.62 & -.23 & &. .29 & -1.44 & 2.60 & $70 \mathrm{SA}$ & $c 476$ \\
\hline $\mathrm{CA} 79 \mathrm{~A}$ & $x$ & 49.48 & 50.30 & $63.36 x$ & 49.03 & 4.90 & $61.84 x$ & -.45 & & .00 & -1.52 & $1.83 x$ & $70 S A$ & $\operatorname{c479A}$ \\
\hline C479B & $\sigma$ & $50.63 x$ & $51.28=$ & 59.39 & 49.40 & 50.45 & 57.68 & $-1 \cdot 23$ & $\mathbf{x}$ & .083 & -1.71 & 2.68 & $705 A$ & $C 479 B$ \\
\hline CABO & $\theta$ & 49.66 & 50.76 & 59.01 & 49.21 & 50.30 & 57.31 & .45 & &. .46 & -1.70 & 2.52 & 7081 & $c 480$ \\
\hline$C 481$ & 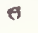 & 49.81 & 50.84 & 59.34 & 49.56 & 50.54 & 57.87 &. .26 & & -.30 & -1.47 & 2.54 & $70 \mathrm{KS}$ & $C 481$ \\
\hline$C 483$ & $\mathrm{x}$ & 49.56 & 50.80 & 59.19 & 49.13 & 50.19 & $57 \cdot 53$ & -.43 & & -.60 & -1.66 & $3.08 x$ & $70 \mathrm{ZF}$ & $C 483$ \\
\hline C496A & $\theta$ & 49.06 & 50.16 & 58.72 & 49.14 & 50.20 & 57.58 & .09 & - & .04 & -1.14 & 2.72 & $70 \mathrm{GE}$ & $C 4961$ \\
\hline C499C & $\mathrm{x}$ & 45.88 & 51.16 & 59.37 & 49.59 & 50.63 & 57.71 & .29 & &. .53 & -1.66 & $3.65 x$ & $70 B L$ & $\mathrm{C} \& \mathrm{SQC}$ \\
\hline $\operatorname{csos}$ & 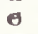 & 48.85 & 49.86 & 58.39 & 48.49 & 49.44 & 56.79 & -.37 & & -.42 & -1.60 & 2.61 & $70 G E$ & C50 3 \\
\hline C50B & $\boldsymbol{\theta}$ & 49.05 & 50.12 & 58.59 & 49.13 & 50.16 & 57.42 & .08 & $\bullet$ & .03 & -1.18 & 2.76 & $7 O C E$ & $\csc 8$ \\
\hline C51 1 & $\theta$ & 49.58 & 50.68 & 59.27 & 49.22 & 50.28 & 57.66 &. .36 & & -.40 & -1.61 & 2.60 & $70 \mathrm{SA}$ & C51 1 \\
\hline C521A & $x$ & 45,83 & 50.99 & 59.43 & 48.87 & 49.96 & 57.09 & -.95 & $x$ & -1.03 & -2.34 & $3.05 x$ & $70 \mathrm{CA}$ & $\operatorname{c5} 21 \mathrm{~A}$ \\
\hline $\operatorname{cs} 21 \mathrm{~B}$ & $x$ & $50.42 x$ & $51.53 \%$ & 59.71 & $50.66 x$ & $51.73 x$ & $58.52=$ & .24 & $x$ & .20 & -1.19 & $3.17 x$ & $70 S A$ & $\mathrm{Cs} 21 \mathrm{~B}$ \\
\hline C522 & $\theta$ & 49.81 & $51.35 \cdot$ & $60.11=$ & 49.02 & 50.57 & 58.10 &. .78 & 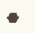 & -.78 & -2.01 & $2.41=$ & $70 S A$ & C522 \\
\hline C524 & $\theta$ & 49.42 & 50.46 & 58.92 & 49.19 & 50.20 & 57.45 & .23 & & -.26 & -1.47 & 2.61 & TOGE & c524 \\
\hline $\operatorname{c5} 28$ & $\sigma$ & 45.75 & 50.86 & 59.63 & 49.47 & 50.61 & 58.01 &. .28 & &. .25 & -1.62 & 2.56 & 7094 & C528 \\
\hline c531 & 8 & 49.22 & 50.28 & 58.79 & 48.94 & 49.97 & 57.31 & -.27 & & .32 & -1.49 & 2.55 & $700 E$ & $\operatorname{c531}$ \\
\hline C532 & 6 & 49.25 & 50.29 & 58.84 & 48.71 & 49.70 & 57.03 & -.54 & &. .59 & -1.81 & 2.67 & $70 G E$ & $\operatorname{c532}$ \\
\hline C534 & $\sigma$ & 49.87 & 51.01 & 55.71 & 49.81. & $50.90=$ & $58.42=$ & -.06 & &. .11 & -1.29 & 2.66 & $70 S A$ & C534 \\
\hline c536 & $\theta$ & 49.77 & 50.86 & 59.34 & 49.55 & 50.60 & 57.90 & -.22 & &. .27 & -1.44 & 2.58 & 7ors & c536 \\
\hline C5\&O & $\theta$ & 49.43 & 50.55 & 58.97 & 49.11 & 50.18 & 57.4 & -.32 & & -.37 & -1.53 & 2.59 & $70 G E$ & c540 \\
\hline $\operatorname{cs} 45$ & 6 & 49.01 & 50.11 & 58.44 & 48.58 & 49.63 & 56.78 &. .43 & & -.47 & -1.65 & 2.58 & 7 OSA & c545 \\
\hline $\operatorname{cs} 48$ & $\boldsymbol{\theta}$ & 48.59 & 50.00 & 58.34 & 48.84 & 49.74 & 56.97 & -.15 & &. .25 & -1.36 & 2.82 & $70 \mathrm{SB}$ & C548 \\
\hline $\operatorname{c5} 49$ & $\theta$ & 49.23 & 50.27 & 58.84 & 48.81 & 49.80 & 57.15 & -.42 & & -.47 & -1.69 & 2.67 & TOGE & $c 549$ \\
\hline C552 & a & 49.34 & 50.43 & 58.58 & 49.12 & 50.16 & 57.11 & .23 & &. .27 & -1.47 & 2.62 & $70 \mathrm{EN}$ & C552 \\
\hline C612 & $\boldsymbol{\sigma}$ & 49.06 & 50.13 & 58.65 & 48.98 & 50.02 & $57 \cdot 32$ & -.08 & & -.12 & $-1 \cdot 33$ & 2.64 & 70GE & C612 \\
\hline 0613 & 6 & 49.77 & 50.87 & 59.58 & 49.54 & 50.59 & 58.14 &. .23 & &. .29 & -1.43 & 2.58 & $705 A$ & C613 \\
\hline C627 & $\theta$ & 45.89 & 51.04 & 59.67 & 49.49 & 50.54 & 58.04 & -.40 & & -.51 & -1.63 & 2.79 & $70 S A$ & C627 \\
\hline C629 & $\theta$ & 49.44 & 50.51 & 58.67 & 49.03 & 50.04 & 56.98 & -.41 & & -.47 & -1.69 & 2.72 & $70 \mathrm{HN}$ & C629 \\
\hline C630 & $\theta$ & 49.31 & 50.40 & 58.86 & 49.14 & 50.18 & 57.46 &. .16 & &. .22 & -1.40 & 2.64 & $70 \mathrm{Ks}$ & c630 \\
\hline
\end{tabular}



COLOR - COLOR DIFEBEENCE

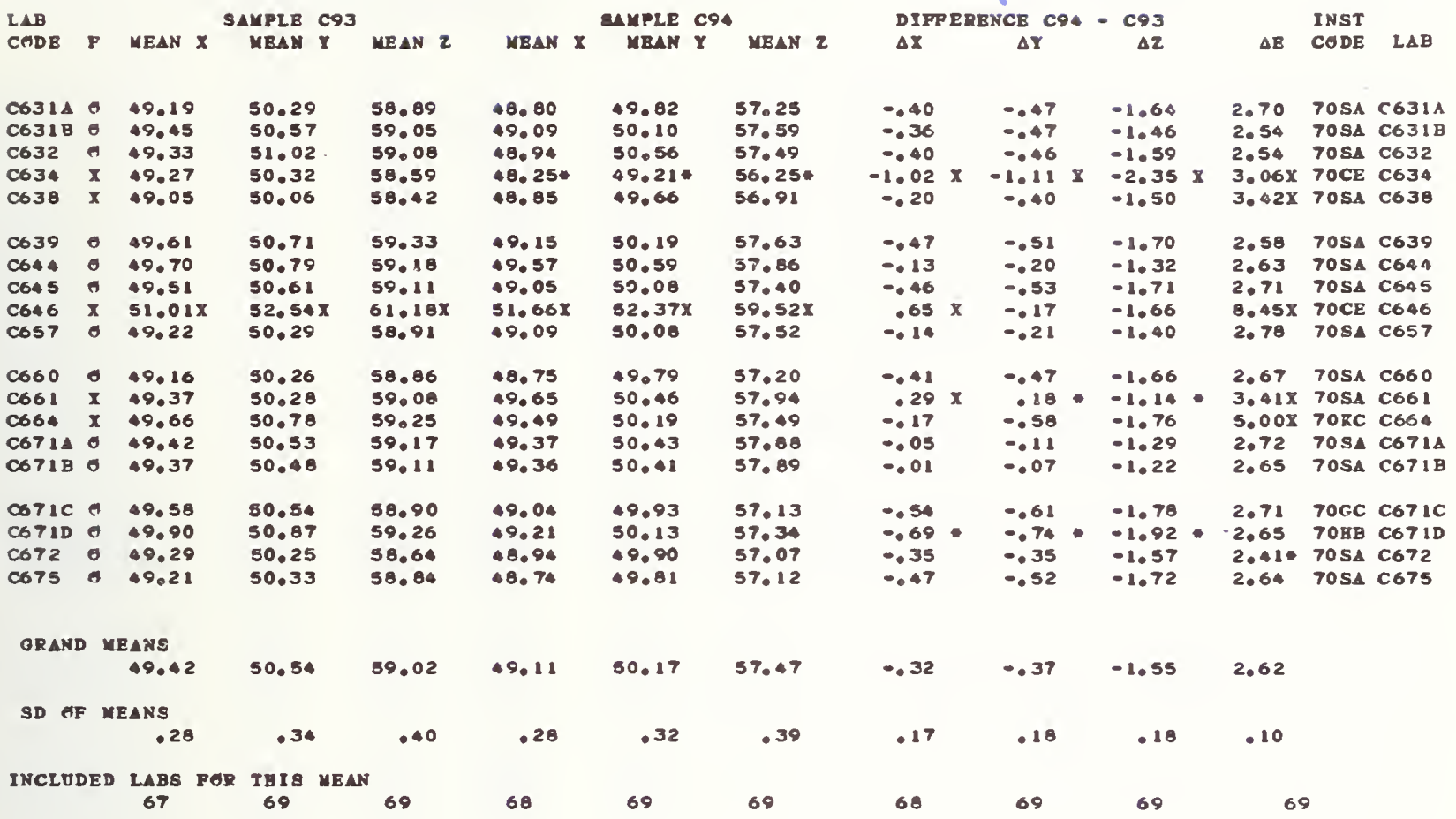


IA

C105 $\odot \quad 35.21$

c121 35.07

C122 35.42

C148 935.56

C 150 o 35.42

C152 535.25

c166 ब 35.45

C183 35.00

C213 35.27

C223 35.31

C230 35.50

C241 35.62

C256 X 35.22

c259 $\theta \quad 35.44$

C262 O 35.40

C285 $6 \quad 35.41$

C291 35.20

C317 35.14

C320 $6 \quad 35.06$

C325 135.07

C340 ค 35.67

C352 35.39

C 356 ब 35.11

C 380 ब 35.38

C382 35.63

$\mathrm{C} 402 \Theta 35.36$

C417A $\theta$ 35.24

C417B X 35.32

C420 35.66

C427 35.17

C44O 35.56

$\mathrm{C} 442$ e 35.14

C454 e 34.94

$\mathrm{C} 456$ \% 35.45

C458 35.34

C475 35.00

C477 35.27

$\mathrm{C} 494$ 35.70

$\mathrm{C}_{496 \mathrm{~B}} \times 34.29 \mathrm{X}$

C459A 35.45

C459B X 35.39

C506 ल 35.47

C517 $94.77 \circ$

C5 38 o $36.75 x$

C542X $33.97 x$

C543@ 35.65

C546A $83.64 X$

C546B $\theta 34.59$

C567 35.60

C576 $637.03 \mathrm{X}$

C585 85.47

0600 35.11

C620 a 35.54

$\mathrm{C} 633$ ○ $35.2 \mathrm{C}$

0647 o 35.08

$\cos 4$ o $34.42 x$

$\begin{array}{lll}6655 & 6 & 34.92 \\ 6662 & 35.84\end{array}$

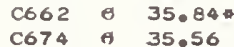

0677 \& 34.96
SAMPIE C9I

$\begin{array}{ll}8.36 & 14.34 \\ 8.96 & 14.44 \\ 9.17 & 14.18 \\ 8.96 & 14.12 \\ 8.80 & 14.00 \\ 8.78 & 14.09 \\ 8.65 & 14.14 \\ 8.54 & 14.18 \\ 8.54 & 13.49 \\ 8.46 & 14.00\end{array}$

$8.80 \quad 14.28$

$9.03 \quad 14.34$

$9.51 \quad 13.91$

$8.65 \quad 14.10$

\subsection{9}

8.96

9.01

8.19

8.43

8.47

8.75

0.29

8.86

8.09 .

8.68

8.80

9.05

9.00

9.30

8.61

9.14

8.98

8.86

9.14

8.74

9.19

9.02

$.21 x$

8. 76

$4.43 x$

9.22

8.47

10. $79 \mathrm{X}$

9.10

8.63

S. 50

9.15

8.91

9.10

9.10

$9.60 \%$

$8.08=$

S. 17

8. 75

14. $C 7$

14.29

14.03

13.82

14.03

14.20

14.04

14.21

13.95

14.07

14.03

14.26

13.95

14.33

14.17

14.11

13.89

14.08

14.09

13.47 .

13. 78

14.08

$.86 x$

13.53

14.08

14.09

13.57 .

14.49

13.66

14.27

14.02

14.12

14.45

14.41

14.01

$8.01 \mathrm{x}$

13. 72

14.19

$17.01 x$

\subsection{3}

13.87

8.63

14.07

9.04

14.02

13.82

13.86
SAMPLB C92

MEAN L MBAN A MEAN B
DIFFERENCE C92 - C91

$\triangle A$

$\Delta B$

INST

$\triangle E$ CEDE LAB

35.11
34.97
35.32
35.46
35.32
35.10
35.40
34.90
35.07
35.22
35.56
35.52
34.74
35.29
35.29
35.21
35.50
35.19
34.76
34.85
35.73
35.09
34.90
35.43
35.33
35.41
35.19
34.84
35.51
35.42

$\begin{array}{ll}9.06 & 14.39 \\ 9.67 & 14.24 \\ 9.88 & 14.12 \\ 9.71 & 14.22 \\ 9.61 & 14.01\end{array}$

.10
.10
$\because 10$
-10
.11
.15
.05
.10
.20
.10

.70
.70
.70
.75
.81
.75
.85
.76
.71
74

.05

$.71708 \mathrm{Cl} 105$

$9.53 \quad 14.04$

$9.51 \quad 14.04$

$9.30 \quad 14.17$

9.20

13.95

.06
.10
.148
.15
.11
.120
.30
.05
.30
.022

.60

$9.40 \quad 13.99$

$10.36 \cdot 14.06$

$11.05 \times \quad 14.03$

$9.32 \quad 14.09$

$9.49 \quad 14.27$

$9.56 \quad 14.04$

9.7113 .93

$9.04 \quad 13.92$

$9.09 \quad 13.91$

9.5614 .30

$9.99 \quad 14.04$

$9.52 \quad 14.16$

$9.41 \quad 14.08$

$9.57 \quad 14.14$

$9.87 \quad 14.32$

$9.70 \quad 14.00$

35.42

10.00

14.00
14.19

35.44

34.84

34.73

35.37

9.3314 .08

9.84

13.92

9.6214 .14

35. 17

35. 14

35.65

34.410

$9.43 \quad 13.48$

$9.90 \quad 13.73$

$9.02 \quad 13.88$

$9.47 \quad 14.03$

.06
.30
.20
.05

.8

.85

.06

.020
.05

7470 HN C121

$7170 \mathrm{BX}$ C 122

.76 70 HA C148
.82 70HA C150

.10
.01

$.7770 \mathrm{HA} \mathrm{C152}$

.86 7OHA C 166

- $76 \quad 70 \mathrm{BA} C 183$

. $7470 \mathrm{BM}$ C213

. 75 7ОRA C223

.6770 sc c230

$.8170 \mathrm{AA} \mathrm{C241}$

- $99 \times 70 \mathrm{HM} \mathrm{C256}$

. 83 70Ha c25s

$.80 .20 \cdot .85 \quad 70 \mathrm{BA} \mathrm{C285}$

$.60 \quad .25 . .72$ 70RA C291

$.70 \quad-10 \quad .71708 \mathrm{C} 317$

$.85-.10 \quad .91 .7014 c 320$

35.34

35.27

34.62.

$36.75 x$

$\begin{array}{ll}9.66 & 14.08 \\ 9.92 & 13.99\end{array}$

$9.17 \quad 13.99$

$11.40 \mathrm{X} \quad 13.57$.

$\begin{array}{ll}1.40 x & 14.49 \\ 9.59 & 13.79\end{array}$

35.40

$33.54 x$

9.45

35.09

$10.26=$

14.34

9.86

14.12

14.02

35.56
$36.99 X$

9.68

14.39

35.28

$9.81 \quad 13.95$

35.11

10.41

8.69.

$8.01 x$

35.27

9.91

13.64

$34.46=$

34.72

35. 89.

35.61

34.96

9.94

$16.95 x$

.62
.80
.71
.65
.80
.73
.78
.82
.70
.70

.12

$.6370 \mathrm{HA}$ c340

. 8670 EA C352

.7370 मM C356

$\begin{array}{llll}.05 & -.05 & .66 & 70 \mathrm{EA} \mathrm{C380}\end{array}$

$\begin{array}{llllll}.05 & .73 & .02 & .74 & 70 \mathrm{HA} & \mathrm{C} 402\end{array}$

.05
$.04-.78$
.0 .05

$-15$

.25

.73

.12 

a, b SPACE ANALYSIS, ADJUSTED
colOR - COLOR DIFPERENCE

$1+$

C105 o 71.83

C121 o 71.00

$\mathrm{Cl} 22$ o 71.59

C148 1471.67

C150 a 71.84

C152 71.66

C166 त 71.60

c183 o $71.15 x$

C213 71.56

C223 91.81

C230 71.66

C241 o 71.75

C256 071.70

c259 71.74

C262 क 71.90

C285 o 71.83

C291 71.77

C317 \& 71.60

C320 $\mathrm{C} 71.82$

C325 71.78

C340 72.08

C352 71.99

C356 71.63

C380 72.02

C382 71.57

$\mathrm{C} 402$ 71.85

C417A 971.71

C417B A 71.53

C420 क 71.88

C427 H 71.85

$\mathrm{C} 440$ क 72.01

$\mathrm{C} 442 \odot 71.50$

C454 A 71.78

C456 A 71.79

C458 A 71.73

C475 71.89

$477 \sigma \quad 71.80$
0.98

C49 क 71.65

C496B X $70.34 X$

C499A \& 71.56

C459B a 71.64

C506 $\rightarrow 71.74$

C517 $\theta 71.03 x$

C538 o 73.19x

C542 $70.29 x$

C543 72.03

C546A $70.65 x$

C546B o 71.49

C567 6 71.88

C576 o 72.23.

. $.92 x$

$.064 \quad 1.07$

.12

. .45

85

.006

.17

.01
-.012

. .33

.05

.25

.16

.06

$.20 \%$

.15

.032

$-.35$

.07

. .24

.32

.17

$\because 32$

. .27

-.34
-.2

$-24$

.25
. .30

.25

$-.09$

$-.26$

$-.25$

$\because 24$

.20

$-13$

.19

.60 .

.20

.25

.08

$2.30 x$

.17

-.41
. .24

.22

. .22

0

.93

.65

.97

1.10

.98

.83
1.03

.96

1.00

1.07
.92

.05

.82

-88

.99

.97

1.02

.95

1. 05

.90

.89
1.06

00

.99
.74
.84

.84
1.00

.68

.77

71.69

1.49
1.07

$70.15 x$

SAMPLB C94

MBAN I MBAK A MEAN B

DIFPEREINCE C94 - $\mathrm{C9} 3$

$\Delta A$

$\Delta \mathrm{B}$

INST

$\triangle E$ CODE LAH

71.15

71.62

71.51

71.40

$71 \cdot 25$

71.29

1.65

71.59

1.04

71.61

$71 \cdot 40$

71.46

71.72

71.32

71.52

71.50

$71.09 \%$

71.58

71.49

71.30

71.59

$1.05 \quad 71.74$

$1.00 \quad 71.39$

$.6070 .63 x$

. $.13 x \quad 72.79 x$

$69.89 x$

1.08

.64
1.00

1. 06

71.67

$70.34 x$

71.29

$71 \cdot 51$

.054.

1.97

2.13

- 13

1.84

.06

.12
.10

.22

. .21

.39

-.04
-.02

.02
.16

$-12$

.25
.10

$-12$

.03

.12

.32

$-.17$

-12
-.27

.20

.08

$\because 10$

.05
. .14

.016

.09

$-18$

$-113$

.10

.06

.03
.01
.000

.24

.10

.15

2. $40 x$

-33*

.22

.24
. .11

.23

.17

1.04

$-31$

.012

. .37

. .23

$-5.94 x$

71.60

1.436

71.72

.18

- 08

$-.25$

. .08
.21

1.91

1.93
1.58

1.58
1.79

1.91

2. 02

1.98
1.75

2.03

.30

$\because 45$
$\because 10$

-10
.05

.017

.10

$-10$

.10

.06

.90

.99

.99

1.00

.15

.20

-.27
-.30

.24

.00

.05
.09

.10

.13

$-.37$

.10

.38

.15
.26

12
.20

$-20$

$-14$

.09

.99

.99

- 93

- 90

.93

1. 59

2.02

1.82

1.84

1.74

1. 92

1. 86

1. 92

$.10 \%$

$.45 \times 1.00$

.10
.15

.20

$-30$

$-36$

-.36
-.50

.50
.$\quad 30$

.35

1.98

1.68

1.88

1.78

2.0

1.87

1.64
1.72

1. 96

1.74

1. 80

1. 88

1.50
2.07

2.05 
NBS VALUES FOR SPECTRAL REFLECTANCE 45/0 REFLECTANCE FACTOR

SAMPLES C92, C94, W14

\begin{tabular}{|c|c|}
\hline WAVELENGTH $(\mathrm{nm})$ & C92 \\
\hline $\begin{array}{l}380 \\
390 \\
400 \\
410 \\
420 \\
430 \\
440 \\
450 \\
460 \\
470 \\
480 \\
490 \\
500 \\
510 \\
520 \\
530 \\
540 \\
550 \\
560 \\
570 \\
580 \\
590 \\
600 \\
610 \\
620 \\
630 \\
640 \\
650 \\
660 \\
670 \\
680 \\
690 \\
700 \\
710 \\
720 \\
730 \\
740 \\
750 \\
760 \\
770\end{array}$ & $\begin{array}{l}.0427 \\
.0452 \\
.0472 \\
.0481 \\
.0487 \\
.0492 \\
.0498 \\
.0504 \\
.0510 \\
.0517 \\
.0528 \\
.0546 \\
.0584 \\
.0673 \\
.0780 \\
.0841 \\
.0887 \\
.0978 \\
.1167 \\
.1437 \\
.1671 \\
.1818 \\
.1888 \\
.1912 \\
.1917 \\
.1911 \\
.1905 \\
.1896 \\
.1887 \\
.1882 \\
.1873 \\
.1870 \\
.1864 \\
.1856 \\
.1849 \\
.1843 \\
.1835 \\
.1826 \\
.1819 \\
.1813\end{array}$ \\
\hline
\end{tabular}

C94 W14

$.1167 \quad .1329$

$.1899 \quad .2255$

$.3202 \quad .4389$

$.4143 \quad .7091$

$.4430 \quad .8584$

$.4607 \quad .8974$

$.4845 \quad .9058$

$\begin{array}{ll}.5020 & .9107\end{array}$

$.5056 \quad .9144$

.5055
.5047

$.5047 \quad .9224$

$.5053 \quad .9256$

$.5095 \quad .9283$

$.5135 \quad .9306$

$.5116 \quad .9322$

$.5062 \quad .9335$

$.5062 \quad .9339$

$.5085 \quad .9340$

$.5029 \quad .9343$

$.5014 \quad .9350$

$.5105 \quad .9362$

$.5177 \quad .9368$

$.5184 \quad .9368$

$.5169 \quad .9363$

$.5162 \quad .9366$

$.5174 \quad .9370$

$.5206 \quad .9372$

$.5279 \quad .9372$

$.5382 \quad .9428$

$.5537 \quad .9305$

$.5746 \quad .9338$

$.6016 \quad .9367$

$.6313 \quad .9365$

$.6632 \quad .9371$

$.6943 \quad .9368$

$.7223 \quad .9378$

.7430

.9375

$\begin{array}{ll}.7576 & .9381 \\ .7680 & .9393\end{array}$

$.7731 \quad .9406$

NBS TRISTIMULUS VALUES

45/0 REFLECTANCE FACTOR

SAMPLES C92, C94, W14

$X, Y, Z$ SPACE

C92

C94

W14

$\begin{array}{rr}X & 13.91 \\ y & 12.26 \\ Z & 6.04\end{array}$

49.90

50.97

57.92

90.93

93.21

106.71

L, a, b SPACE

C92

C94

W14

L $\quad 35.01$

71.39

$-0.18$

96.54

9.68

1.89

$-0.83$

14.27 
SPECTROPHOTCMETRIC CURVES OF COLOR AND

COLOR DIFFERENCE NO. 25 SAMPLES

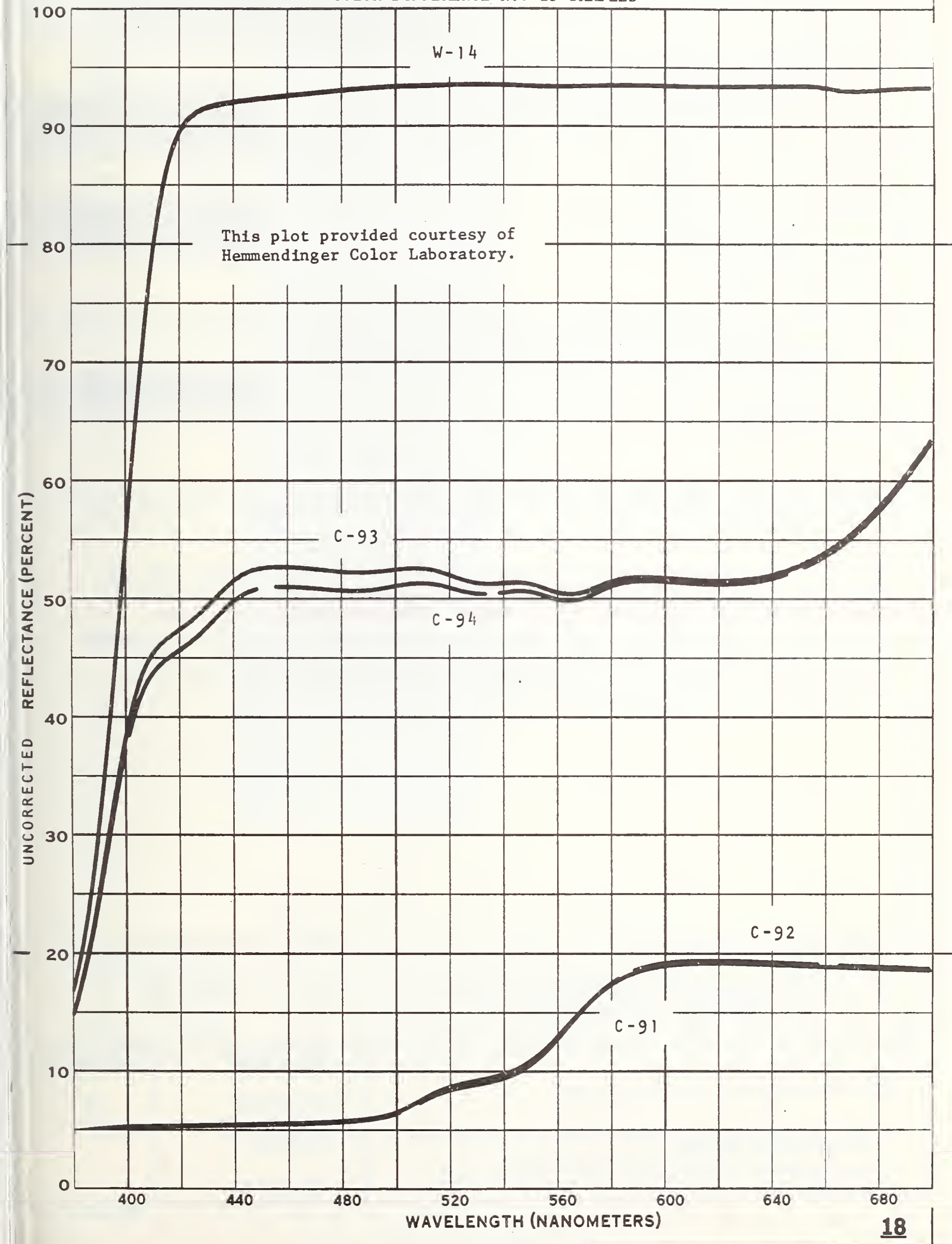


NBS. $114 A$ (REV. 9.78$)$

\begin{tabular}{|c|c|c|}
\hline $\begin{array}{l}\text { U.S. DEPT. OF COMM. } \\
\text { BIBLIOGRAPHIC DATA } \\
\text { SHEET }\end{array}$ & $\begin{array}{l}\text { 1. PUBLICATION OR REPORT NO. } \\
\text { MCCA CRP COIor } 25\end{array}$ & Whoplonts \\
\hline \multicolumn{2}{|c|}{$\begin{array}{l}\text { 4. TITLE AND SUBTITLE } \\
\text { Manufacturers Council on Color and Appearance } \\
\text { Collaborative Reference Program, Color and Appearance } \\
\text { Report No. } 25 \text {, Color and Color Difference }\end{array}$} & 5. Publication Date \\
\hline \multicolumn{2}{|c|}{$\begin{array}{l}\text { 7. AUTHOR(S) } \\
\text { J. Horl ick, T. L. Cummings }\end{array}$} & $\begin{array}{l}\text { 8. Performing Organ. Report No. } \\
\text { NBSIR 79-1368 }\end{array}$ \\
\hline \multirow{2}{*}{\multicolumn{2}{|c|}{$\begin{array}{l}\text { 9. PERFORMING ORGANIZATION NAME AND A } \\
\text { NATIONAL BUREAU OF STANDARDS } \\
\text { DEPARTMENT OF COMMERCE } \\
\text { WASHINGTON, DC } 20234\end{array}$}} & 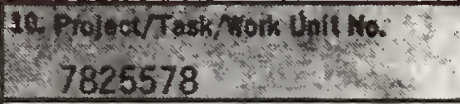 \\
\hline & & 11. Contract/Grant No. \\
\hline \multicolumn{2}{|c|}{$\begin{array}{l}\text { 12. SPONSORING ORGANIZATION NAME AND COMPLETE ADDRESS (streot, City, state, zIP) } \\
\text { Collaborative Testing Services, Inc., } 9241 \text { Wood Glade Drive, } \\
\text { Great Falls, VA 22066; and Manufacturers Council on Color } \\
\text { and Appearance. }\end{array}$} & 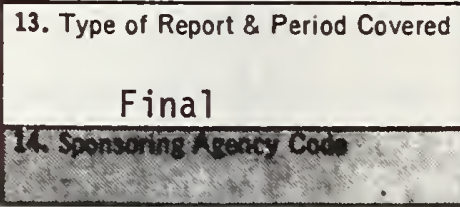 \\
\hline
\end{tabular}

15. SUPPLEMENTARY NOTES

Document describes a computer program; SF-185, FIPS Software Summary, is attached.

16. ABSTRACT (A 200-word or loos factual eummary of most algrificant information. II documont includes a signiflcant bibliography or literature survey, mention it here.)

Collaborative Reference Programs provide participating laboratories with the means for checking periodically the level and uniformity of their testing in comparison with that of other participating laboratories. An important by-product of the programs is the provision of realistic pictures of the state of the testing art. This is one of the periodic reports showing averages for each participant, within and between laboratory variability, and other information for participants and standards committees.

17. KEY WORDS (six to tweive entries; aiphabefical order; copltalize onil the firot letter of the first key word unleos a proper name; eeparated by semicolone)

Collaborative reference program; Color; Laboratory evaluation; Precision; Reference samples: Testing calibration

18. AVAILABILITY $\square$ Unlimited

XX For Official Distribution. Do Not Release to NTIS

Order From Sup. of Doc., U.S. Government Printing Office, Washington, DC 20402, SD Stock No. SNÖ03-003-

$\square$ Order From National Technical Information Service (NTIS), Springfield, VA. 22161

\begin{tabular}{|l|c|}
\hline $\begin{array}{l}\text { 19. SECURITY CLASS } \\
\text { (THIS REPORT) } \\
\text { UNCLASSIFIED }\end{array}$ & $\begin{array}{c}\text { 21. NO. OF } \\
\text { PRINTED PAGES }\end{array}$ \\
\hline $\begin{array}{l}\text { 20. SECURITY CLASS } \\
\text { (THIS PAGE) }\end{array}$ & 23 \\
UNCLASSIFIED & 22. Price \\
\hline
\end{tabular}

